\title{
Kähler-Poisson Algebras
}

\section{Ahmed Al-Shujary}




\title{
Kähler-Poisson algebras
}

\author{
Ahmed Al-Shujary
}

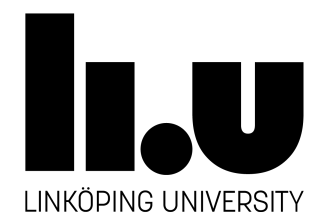

Department of Mathematics

Linköping, 2020 
Linköping Studies in Science and Technology. Dissertations No. 2044

\section{Kähler-Poisson algebras}

Copyright (C) Ahmed Al-Shujary, 2020

Department of Mathematics

Linköping University

SE-581 83 Linköping, Sweden

Email: ahmed.al-shujary@liu.se

ISSN 0345-7524

ISBN 978-91-7929-909-5

Printed by LiU-Tryck, Linköping, Sweden, 2020 


\section{Abstract}

In this thesis, we introduce Kähler-Poisson algebras and study their basic properties. The motivation comes from differential geometry, where one can show that the Riemannian geometry of an almost Kähler manifold can be formulated in terms of the Poisson algebra of smooth functions on the manifold. It turns out that one can identify an algebraic condition in the Poisson algebra (together with a metric) implying that most geometric objects can be given a purely algebraic formulation. This leads to the definition of a Kähler-Poisson algebra, which consists of a Poisson algebra and a metric fulfilling an algebraic condition. We show that every KählerPoisson algebra admits a unique Levi-Civita connection on its module of inner derivations and, furthermore, that the corresponding curvature operator has all the classical symmetries. Moreover, we present a construction procedure which allows one to associate a Kähler-Poisson algebra to a large class of Poisson algebras. From a more algebraic perspective, we introduce basic notions, such as morphisms and subalgebras, as well as direct sums and tensor products. Finally, we initiate a study of the moduli space of Kähler-Poisson algebras; i.e for a given Poisson algebra, one considers classes of metrics giving rise to non-isomorphic Kähler-Poisson algebras. As it turns out, even the simple case of a Poisson algebra generated by two variables gives rise to a nontrivial classification problem. 


\section{Sammanfattning}

I denna avhandling introduceras Kähler-Poisson algebror och deras grundläggande egenskaper studeras. Motivationen till detta kommer från differentialgeometri där man kan visa att den metriska geometrin för en Kählermångfald kan formuleras i termer av Poisson algebran av släta funktioner på mångfalden. Det visar sig att man kan identifiera ett algebraiskt villkor i en Poissonalgebra (med en metrik) som gör det möjligt att formulera de flesta geometriska objekt på ett algebraiskt vis. Detta leder till definitionen av en Kähler-Poisson algebra, vilken utgörs av en Poissonalgebra och en metrik som tillsammans uppfyller ett kompatibilitetsvillkor. Vi visar att för varje Kähler-Poisson algebra så existerar det en Levi-Civita förbindelse på modulen som utgörs av de inre derivationerna, och att den tillhörande krökningsoperatorn har alla de klassiska symmetrierna. Vidare presenteras en konstruktion som associerar en Kähler-Poisson algebra till varje algebra i en stor klass av Poissonalgebror. Ur ett mer algebraiskt perspektiv så introduceras flera grundläggande begrepp, såsom morfier, delalgebror, direkta summor och tensorprodukter. Slutligen påbörjas en studie av modulirum för Kähler-Poisson algebror, det vill säga ekvivalensklasser av metriker som ger upphov till isomorfa Kähler-Poisson strukturer. Det visar sig att även i det enkla fallet med en Poisson algebra genererad av två variabler, så leder detta till ett icke-trivialt klassificeringsproblem. 


\section{Acknowledgments}

First of all, I am very grateful to my main supervisor, Joakim Arnlind, for many interesting discussions, thoughtful comments, and your patience and support. I would like to express my deep gratitude to my co-supervisor Milagros Izquierdo, for ideas, discussions and for useful comments in the writing up of this thesis. The department of Mathematics at Linköping University is a very nice place to work at and therefore I would like to thank all of my colleagues, the administration and all the PhD students for making a pleasant working environment. I would like to thank the Ministry of Higher Education and Scientific Research in Iraq for the financial support.

I am grateful to my family for their support. Thank you my dear father for your love, courage and prayers. My late mother I will never forget you. Thank you my wife Marwah and our children Fatima, Narjis and Adam for your encouragement. 


\section{Contents}

Abstract. . . .................... i

Sammanfattning . . . . . . . . . . . . . . . . . . ii

Acknowledgments . . . . . . . . . . . . . . . . . . . .

Contents.......................... vii

$\begin{array}{lll}1 & \text { Introduction } & 1\end{array}$

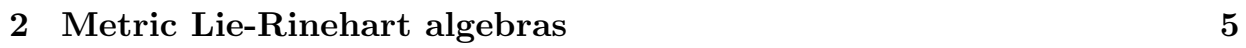

$\begin{array}{lll}3 & \text { Kähler-Poisson algebras } & 9\end{array}$

3.1 Poisson algebras $\ldots \ldots \ldots \ldots \ldots$

3.2 Kähler-Poisson algebras . . . . . . . . . . . . . . . . . . . 11

3.3 Homomorphisms of Kähler-Poisson algebras . . . . . . . . . . . . 17

3.4 Subalgebras of Kähler-Poisson algebras . . . . . . . . . . . . . . . 23

3.5 Direct sums and tensor products of Kähler-Poisson algebras . . . . 25

3.6 Construction of Kähler-Poisson algebras $\ldots \ldots \ldots \ldots . \ldots 27$

\begin{tabular}{|lll}
4 & Curvature and isomorphism classes & 29
\end{tabular}

4.1 Levi-Civita connections and curvature . . . . . . . . . . . . . 29

4.2 Properties of isomorphisms . . . . . . . . . . . . . . . . 31

$4.3 \quad$ Moduli spaces for a class of Kähler-Poisson algebras _ . . . . . . . 32

\begin{tabular}{ll}
\hline Paper I & 39
\end{tabular}

Kähler-Poisson algebras

Joakim Arnlind and Ahmed Al-Shujary. J. of Geom. and Phys. 136, 2019.

Paper II

Morphisms, direct sums and tensor products of Kähler-Poisson algebras

Ahmed Al-Shujary.

Paper III

On moduli spaces of Kähler-Poisson algebras over rational functions in two variables Ahmed Al-Shujary. 


\section{1 - Introduction}

Symplectic manifolds (in particular cotangent bundles) arise naturally as the phase space of classical mechanics. A symplectic manifold is a pair $(M, \omega)$ where $M$ is an even-dimensional manifold and $\omega$ is a symplectic form on $M$. On a symplectic manifold, the symplectic form induces a Poisson bracket on the smooth functions (see Section 3.1). Poisson manifolds are generalizations of symplectic manifolds to odd-dimensional manifolds. Algebraic characterizations of Poisson manifolds are responsible for the natural appearance of objects which are both of geometric and algebraic nature. The techniques involved in Poisson geometry vary a lot, and include not only differential geometric and algebraic ones, but also techniques arising from physics. For instance, a classical mechanical system in the Hamiltonian formalism is described by the triple $(M,\{\cdot, \cdot\}, H)$ where $M$ is an even-dimensional manifold called the phase space, $\{\cdot, \cdot\}$ is a Poisson bracket, given by a symplectic or Poisson structure on $M$ and $H$ is a smooth function on $M$, called the Hamiltonian. Many authors have studied the geometric and algebraic properties of symplectic and Poisson manifolds (see e.g. [8, 12, 13, 16, 19]). For instance, Kontsevich [16], has shown that any finite dimensional Poisson manifold $M$ admits a canonical deformation quantization by establishing a correspondence between the set of isomorphism classes of deformations of the algebra of smooth functions and the set of equivalence classes of formal Poisson structures on $M$.

However, metric aspects of Poisson manifolds have not been investigated to the same extent. The study of metric aspects was motivated by the results in [1, 2], where the differential geometry of embedded (almost) Kähler manifolds $\Sigma$ was formulated in terms of the Poisson structure of the algebra of functions of $\Sigma$. In the following, we set out to find structures that resemble Poisson algebras of smooth functions on Kähler manifolds. A Kähler manifold is a manifold with three mutually compatible structures: a complex structure, a Riemannian structure, and a symplectic structure. Let $M$ be a complex manifold with corresponding complex structure $J$. We say that a Riemannian metric is compatible with $J$ if

$$
g(J X, J Y)=g(X, Y)
$$

for all vector fields $X, Y$ on $M$. A complex manifold together with a compatible Riemannian metric is called a Hermitian manifold. See e.g. 28] for more details on complex manifolds. Let $M$ be Hermitian manifold with complex structure $J$ and compatible Riemannian metric as in 1.1. The alternating 2 -form defined by $\omega(X, Y)=g(J X, Y)$ is called the associated Kähler form. Conversely, one can retrieve $g$ from $\omega$ by defining $g(X, Y)=\omega(X, J Y)$. We say that $g$ is a Kähler metric and that $M$ (together with $g$ ) is a Kähler manifold if $\omega$ is closed [18. Thus, a Kähler manifold is a symplectic manifold with respect to the Kähler form. Kähler manifolds and their properties have been extensively studied, see e.g. [8], [19] and 27. 
In this thesis we will develop algebraic analogues of Kähler manifolds by using the language of Lie-Rinehart algebras, which we will extend to a metric setting. As shown in [1], the Riemannian geometry of embedded almost Kähler manifolds can be reformulated entirely in terms of the Poisson algebra of smooth functions. In particular, one obtains Poisson algebraic expressions for geometric quantities by using the algebra generated by the embedding coordinates. We observe that on a Kähler manifold $(\Sigma, g)$ isometrically embedded in the Riemannian manifold $(M, \bar{g})$, the compatibility between the metric and the symplectic form (and, hence, the Poisson structure) implies that

$$
\sum_{i, j, k, l=1}^{m}\left\{f_{1}, x^{i}\right\} \bar{g}_{i j}\left\{x^{j}, x^{k}\right\} \bar{g}_{k l}\left\{x^{l}, f_{2}\right\}=-\left\{f_{1}, f_{2}\right\}
$$

for all $f_{1}, f_{2} \in C^{\infty}(\Sigma)$, where $x^{1}, \ldots, x^{m}$ is a set of smooth functions providing an isometric embedding of $\Sigma$ into $M$. Note that this situation is generic in the sense that any Riemannian manifold can be isometrically embedded in Euclidean space 22. Surprisingly, it turns out that if one imposes the above relation in an abstract Poisson algebra (i.e. not necessarily the function algebra of a manifold), many classical results in Riemannian geometry can be worked out in a purely algebraic setting.

In this thesis, we begin to study metric aspects of Poisson algebras by defining Kähler-Poisson algebras as algebraic analogues of algebras of functions on Kähler manifolds. We show that any Poisson algebra, fulfilling a "Kähler condition" similar to 1.2 , enjoys many properties similar to those of the algebra of smooth functions on an almost Kähler manifold, opening up for a more metric treatment of Poisson structures. Moreover, we show that one may associate a Kähler-Poisson algebra to every algebra in a large class of Poisson algebras. Furthermore, we prove the existence of a unique Levi-Civita connection on the module generated by the inner derivations, and show that the curvature operator has all the classical symmetries. In parallel with the more geometric results, we also explore basic algebraic properties and structures related to Kähler-Poisson algebras. In particular, we find appropriate definitions of morphisms, subalgebras, direct sums and tensor products of Kähler-Poisson algebras.

To better understand the concept of morphism, we construct several examples that illustrate when two Kähler-Poisson algebras are isomorphic. The possibility of choosing different sets of generators for a finitely generated algebra is investigated, as well as the implications for the structure of the Kähler-Poisson algebra. In this context, a natural question arises: Given a Poisson algebra, in how many different (non-isomorphic) ways can one add an extra metric structure to obtain a KählerPoisson algebra? Using the algebraic results obtained in this thesis, we initiate a study of "moduli spaces", i.e. the study of isomorphism classes of Kähler-Poisson algebras. Even in the simple setting of an algebra generated by two elements, this problem turns out to be non-trivial.

The thesis is composed of three papers. Let us now briefly review the contents of Paper I, II and III. In Paper I [3], we introduce the concept of Kähler-Poisson algebras as analogues of algebras of smooth functions on Kähler manifolds, and explain how the theory of Lie-Rinehart algebras can be extended to include metric 
aspects. We prove that Kähler-Poisson algebras are metric Lie-Rinehart algebras, which implies that the theory of Lie-Rinehart algebras can be applied. Furthermore, we show that the "Kähler-Poisson condition", being the crucial identity in the definition of Kähler-Poisson algebras, allows for an identification of geometric objects in the Poisson algebra which share important properties with their classical counterparts. For instance, it is shown that there exists a unique Levi-Civita connection on the module generated by the inner derivations of the Kähler-Poisson algebra, and that the curvature operator has all the classical symmetries. Moreover, starting from a large class of Poisson algebras, we show that every algebra has an associated Kähler-Poisson algebra constructed as a localization.

In Paper II 5, the concept of morphisms of Kähler-Poisson algebras is introduced and their algebraic properties are studied. We illustrate with several examples when two Kähler-Poisson algebras are isomorphic; for instance, by taking algebras $\mathcal{A}$ and $\mathcal{A}^{\prime}$, where $\mathcal{A}$ is finitely generated algebra, $\mathcal{A}^{\prime}=\mathcal{A}$ and considering different set of generators for the Kähler-Poisson algebra structures of $\mathcal{A}$ and $\mathcal{A}^{\prime}$. Moreover, we use the concept of morphism to define subalgebras of KählerPoisson algebras, and introduce direct sums and tensor products of Kähler-Poisson algebras together with their basic properties.

In Paper III [6], we initiate a study of moduli spaces of Kähler-Poisson algebras defined over rational functions in two variables. Starting from a Poisson algebra $\mathcal{A}$, it is interesting to ask the following question: How many non-isomorphic Kähler-Poisson algebras can one construct from $\mathcal{A}$ ? This amounts to the study of a "moduli space" for Kähler-Poisson algebras, in analogy with the corresponding problem for Riemannian manifolds, where one consider metrics giving rise to non-isometric Riemannian manifolds. For certain types of metrics, we completely characterize the isomorphism classes of Kähler-Poisson algebras. To prove these statements, we make use of the results obtained in Paper II. Furthermore, several sufficient conditions for the existence of isomorphisms are derived, and a number of examples are given illustrating that seemingly different metrics may give rise to isomorphic Kähler-Poisson algebras.

\section{Outline}

Let us outline the contents of the thesis. In Chapter 2 we recall the first part of Paper I [3] and explain how the theory of Lie-Rinehart algebras can be extended to include metric aspects. In Chapter 3, Kähler-Poisson algebras are defined and the basic algebraic results from Paper I and II are presented. Chapter 4 contains the more geometrical results from Paper I, such as the existence of a Levi-Civita connection, together with the investigation of moduli spaces from Paper III. More precisely, the structure of the thesis is as follows:

- In Chapter 2, we review the concept of Lie-Rinehart algebras and introduce metric Lie-Rinehart algebras.

- In Chapter 3, we introduce the main objects of this thesis, called KählerPoisson algebras, together with their basic properties as well as a construc- 
tion procedure for associating a Kähler-Poisson algebra to a Poisson algebra. Furthermore, morphisms of Kähler-Poisson algebras are defined and we introduce subalgebras, direct sums and tensor products of Kähler-Poisson algebras. Several results are proven showing that these concepts have the properties one expects in an algebraic setting.

- In Chapter 4, the Levi-Civita connection is introduced and we prove that every Kähler-Poisson algebra admits such a connection. Examples are given where the connection, as well as the curvature, is explicitly computed. Moreover, we initiate a study of moduli spaces of Kähler-Poisson algebras defined over rational functions in two variables, and prove that one can describe isomorphism classes of Kähler-Poisson algebras for certain types of metrics. 


\section{2 - Metric Lie-Rinehart algebras}

This chapter is devoted to introducing metric Lie-Rinehart algebras (which is the first part of Paper I [3]), since our approach is quite close to the theory of Lie-Rinehart algebras. A Lie-Rinehart algebra $(\mathcal{A}, \mathfrak{g})$ consists of a commutative algebra $\mathcal{A}$ and a Lie algebra $\mathfrak{g}$ with additional structure which generalizes the interaction between the algebra of smooth functions and the Lie algebra of vector fields on a smooth manifold. We assume that a (commutative) algebra $\mathcal{A}$ is given (corresponding to the algebra of functions), together with an $\mathcal{A}$-module $\mathfrak{g}$ (corresponding to the module of vector fields) which is also a Lie algebra and acts on $\mathcal{A}$ as derivations. For more details and proofs, we refer to [3, 11, 12, 24. Note that, although Lie-Rinehart algebras have been extensively studied, metric aspects have not been considered to the same extent. Our starting point is the definition given by G. Rinehart [26].

Definition 2.1. (Lie-Rinehart algebra). Let $\mathbb{K}$ be a field. Let $\mathcal{A}$ be a commutative $\mathbb{K}$-algebra and let $(\mathfrak{g},[\cdot, \cdot])$ be an $\mathcal{A}$-module which is also a Lie algebra over $\mathbb{K}$. Given a Lie algebra homomorphism $\omega: \mathfrak{g} \rightarrow \operatorname{Der}(\mathcal{A})$, The pair $(\mathcal{A}, \mathfrak{g})$ is called a Lie-Rinehart algebra if

$$
\begin{aligned}
\omega(a \alpha)(b) & =a(\omega(\alpha)(b)) \\
{[\alpha, a \beta] } & =a[\alpha, \beta]+(\omega(\alpha)(a)) \beta,
\end{aligned}
$$

for $\alpha, \beta \in \mathfrak{g}$ and $a, b \in \mathcal{A}$. (In most cases, we will leave out $\omega$ and write $\alpha(a)$ instead of $\omega(\alpha)(a)$.)

Let us point out some immediate examples of Lie-Rinehart algebras.

Example 2.2. Let $\mathcal{A}$ be a commutative algebra and let $\mathfrak{g}=\operatorname{Der}(\mathcal{A})$ be the $\mathcal{A}$ module of derivations of $\mathcal{A}$. As we mentioned before $\operatorname{Der}(\mathcal{A})$ is a Lie algebra with Lie-bracket, defined as

$$
[\alpha, \beta](a)=\alpha(\beta(a))-\beta(\alpha(a)) .
$$

The pair $(\mathcal{A}, \operatorname{Der}(\mathcal{A}))$ is a Lie-Rinehart algebra where $\operatorname{Der}(\mathcal{A})$ acts as derivations.

Example 2.3. Let $\mathcal{A}=C^{\infty}(\Sigma)$ be the algebra of smooth functions on a manifold $\Sigma$, and let $\mathfrak{g}=\chi(\mathcal{A})$ be the $\mathcal{A}$-module of vector fields on $\Sigma$. With respect to the standard action of a vector field as a derivation of $C^{\infty}(\Sigma)$, the pair $\left(C^{\infty}(\Sigma), \chi(\mathcal{A})\right)$ is a Lie-Rinehart algebra.

In differential geometry, consider a smooth manifold $\Sigma$ of dimension $n$. At each point $p \in \Sigma$ there is a vector space $T_{p} \Sigma$, called the tangent space, consisting of all tangent vectors to the manifold at the point $p$. A metric at $p$ is a function $g_{p}\left(X_{p}, Y_{p}\right)$ which takes as inputs a pair of tangent vectors $X_{p}$ and $Y_{p}$ at $p$, and 
produces as output a real number. Globally, a metric maps two vector fields to a function with $g(X, Y)(p)=g_{p}\left(X_{p}, Y_{p}\right)$.

In the following, we will introduce metrics on Lie-Rinehart algebras, as well as an associated Levi-Civita connection.

Definition 2.4. Let $(\mathcal{A}, \mathfrak{g})$ be a Lie-Rinehart algebra and let $M$ be an $\mathcal{A}$-module. An $\mathcal{A}$-bilinear form $g: M \times M \rightarrow \mathcal{A}$ is called a metric on $M$ if it holds that

1. $g\left(m_{1}, m_{2}\right)=g\left(m_{2}, m_{1}\right)$ for all $m_{1}, m_{2} \in M$,

2. the map $\hat{g}: M \rightarrow M^{*}$, given by $\left(\hat{g}\left(m_{1}\right)\right)\left(m_{2}\right)=g\left(m_{1}, m_{2}\right)$, is an $\mathcal{A}$-module isomorphism, where $M^{*}$ denotes the dual of $M$.

We refer to property (2) as the metric being non-degenerate.

Example 2.5. In the definition above let $M=\mathcal{A}$ and let $g(a, b)=a b$, immediately giving that $g(a, b)=g(b, a)$ Moreover, it follows that

$$
\hat{g}(a)(b)=g(a, b)=a b
$$

If $\mathcal{A}$ is unital, choose $b=1$ then $a b=0 \forall b \in \mathcal{A}(=M)$ implies that $a=0$. Hence $g$ is non-degenerate, implying that $g$ is a metric.

Definition 2.6 ([3]). A metric Lie-Rinehart algebra $(\mathcal{A}, \mathfrak{g}, g)$ is a Lie-Rinehart algebra $(\mathcal{A}, \mathfrak{g})$ together with a metric $g: \mathfrak{g} \times \mathfrak{g} \rightarrow \mathcal{A}$.

In Example 2.3. if one choose $M$ to be the sections of a vector bundle over the manifold then we obtain a metric on the vector bundle, i.e. on the set of smooth sections. We proceed by introducing connections on modules over Lie-Rinehart algebras.

Definition 2.7. Let $(\mathcal{A}, \mathfrak{g})$ be a Lie-Rinehart algebra over $\mathbb{K}$ and let $M$ be an $\mathcal{A}$-module. A connection $\nabla$ on $M$ is a map $\nabla: \mathfrak{g} \rightarrow \operatorname{End}_{\mathbb{K}}(M)$ (i.e. $\mathbb{K}$-linear endomorphisms), written as $\alpha \rightarrow \nabla_{\alpha}$, such that

1. $\nabla_{a \alpha+\beta}=a \nabla_{\alpha}+\nabla_{\beta}$

2. $\nabla_{\alpha}(a m)=a \nabla_{\alpha} m+\alpha(a) m$,

for all $a \in \mathcal{A}, \alpha, \beta \in \mathfrak{g}$ and $m \in M$.

Example 2.8. In Example 2.3, if one chooses $M=\mathfrak{g}$ to be the sections of a vector bundle over the manifold $\Sigma$, then any connection on the vector bundle is a connection in the sense of Definition 2.7.

Definition 2.9. Let $(\mathcal{A}, \mathfrak{g})$ be a Lie-Rinehart algebra and let $M$ be an $\mathcal{A}$-module with connection $\nabla$ and metric $g$. The connection is called metric if

$$
\alpha\left(g\left(m_{1}, m_{2}\right)\right)=g\left(\nabla_{\alpha} m_{1}, m_{2}\right)+g\left(m_{1}, \nabla_{\alpha} m_{2}\right)
$$

for all $\alpha \in \mathfrak{g}$ and $m_{1}, m_{2} \in M$. 
Definition 2.10. Let $(\mathcal{A}, \mathfrak{g})$ be a Lie-Rinehart algebra and let $\nabla$ be a connection on $\mathfrak{g}$. The connection is called torsion-free if

$$
\nabla_{\alpha} \beta-\nabla_{\beta} \alpha-[\alpha, \beta]=0
$$

for all $\alpha, \beta \in \mathfrak{g}$.

It is well-known that, on any manifold, there are infinitely many connections. If the manifold is further endowed with a Riemannian metric $g$ then there is a natural choice of connection, called the Levi-Civita connection.

As in differential geometry, one can show that there exists a unique torsion-free and metric connection associated to the Riemannian metric. The first step involves Koszul's formula, which in the setting of Lie-Rinehart algebras is as follows:

Proposition $2.11([3])$. Let $(\mathcal{A}, \mathfrak{g}, g)$ be a metric Lie-Rinehart algebra. If $\nabla$ is a metric and torsion-free connection on $\mathfrak{g}$ then it holds that

$$
\begin{aligned}
2 g\left(\nabla_{\alpha} \beta, \gamma\right)=\alpha( & g(\beta, \gamma))+\beta(g(\gamma, \alpha))-\gamma(g(\alpha, \beta)) \\
& +g(\beta,[\gamma, \alpha])+g(\gamma,[\alpha, \beta])-g(\alpha,[\beta, \gamma])
\end{aligned}
$$

for all $\alpha, \beta, \gamma \in \mathfrak{g}$.

Since the metric is non-degenerate, one can then show the following.

Proposition $2.12([3])$. Let $(\mathcal{A}, \mathfrak{g}, g)$ be a metric Lie-Rinehart algebra. Then there exists a unique metric and torsion-free connection on $\mathfrak{g}$.

The unique connection in Proposition 2.12 will be referred to as the Levi-Civita connection of a metric Lie-Rinehart algebra. We shall recall some of the properties satisfied by a metric and torsion-free connection. The differential geometric proofs go through with only a change in notation needed. We refer to [17, 23. for a nice overview of differential geometric constructions in modules over general commutative algebras.

Furthermore, we define curvature of $\nabla$ as

$$
R(\alpha, \beta) \gamma=\nabla_{\alpha} \nabla_{\beta} \gamma-\nabla_{\beta} \nabla_{\alpha} \gamma-\nabla_{[\alpha, \beta]} \gamma
$$

as well as

$$
\begin{aligned}
R(\alpha, \beta, \gamma) & =R(\alpha, \beta) \gamma \\
R(\alpha, \beta, \gamma, \delta) & =g(\alpha, R(\gamma, \delta) \beta) .
\end{aligned}
$$

Example 2.13 (Continuation of 2.2). If $g$ is a metric on $\operatorname{Der}(\mathcal{A})$ in Example 2.2. then there exists a unique Levi-Civita connection on $\operatorname{Der}(\mathcal{A})$.

Let us also consider the canonical extension of $\nabla$ to multilinear maps $T: \mathfrak{g}^{k} \rightarrow \mathcal{A}$

$$
\left(\nabla_{\beta} T\right)\left(\alpha_{1}, \ldots, \alpha_{k}\right)=\beta\left(T\left(\alpha_{1}, \ldots, \alpha_{k}\right)\right)-\sum_{i=1}^{k} T\left(\alpha_{1}, \ldots, \nabla_{\beta} \alpha_{i}, \ldots, \alpha_{k}\right),
$$


as well as to $\mathfrak{g}$-valued multilinear maps $T: \mathfrak{g}^{k} \rightarrow \mathfrak{g}$

$$
\left(\nabla_{\beta} T\right)\left(\alpha_{1}, \ldots, \alpha_{k}\right)=\nabla_{\beta}\left(T\left(\alpha_{1}, \ldots, \alpha_{k}\right)\right)-\sum_{i=1}^{k} T\left(\alpha_{1}, \ldots, \nabla_{\beta} \alpha_{i}, \ldots, \alpha_{k}\right) .
$$

As in classical geometry, one obtains a generalization of the Bianchi identities.

Proposition 2.14 (3]). Let $\nabla$ be the Levi-Civita connection of a metric LieRinehart algebra $(\mathcal{A}, \mathfrak{g}, g)$ and let $R$ denote corresponding curvature. Then it holds that

$$
\begin{gathered}
R(\alpha, \beta, \gamma)+R(\gamma, \alpha, \beta)+R(\beta, \gamma, \alpha)=0 \\
\left(\nabla_{\alpha} R\right)(\beta, \gamma, \delta)+\left(\nabla_{\beta} R\right)(\gamma, \alpha, \delta)+\left(\nabla_{\gamma} R\right)(\alpha, \beta, \delta)=0
\end{gathered}
$$

for all $\alpha, \beta, \gamma, \delta \in \mathfrak{g}$.

One is also able to derive the classical symmetries of the curvature tensor.

Proposition 2.15 ([3]). Let $\nabla$ be the Levi-Civita connection of a metric LieRinehart algebra $(\mathcal{A}, \mathfrak{g}, g)$ and let $R$ denote corresponding curvature. Then it holds that

$$
\begin{gathered}
R(\alpha, \beta, \gamma, \delta)=-R(\beta, \alpha, \gamma, \delta)=-R(\alpha, \beta, \delta, \gamma) \\
R(\alpha, \beta, \gamma, \delta)=R(\delta, \gamma, \alpha, \beta)
\end{gathered}
$$

for all $\alpha, \beta, \gamma, \delta \in \mathfrak{g}$.

We will show in Chapter 3 that Kähler-Poisson algebras are metric Lie-Rinehart algebras, which implies that the results of this section can be applied; in particular, there exists a unique torsion-free metric connection on every Kähler-Poisson algebra. 


\section{3 - Kähler-Poisson algebras}

In this chapter we shall introduce a type of Poisson algebras that resembles the smooth functions on an (isometrically) embedded almost Kähler manifold. In this way we can develop an analogue of Riemannian geometry for Poisson algebras. Let us start by recalling a few basic properties of Poisson algebras.

\subsection{Poisson algebras}

The concept of a Poisson algebra is classical and appears naturally in Hamiltonian mechanics, and is also central in the study of quantum groups and deformation quantization, (see e.g. [8, 16, 27]). The algebra is named in honour of Siméon Denis Poisson. A Poisson structure on a smooth manifold $\Sigma$ is a Lie bracket on the algebra of smooth functions, which is also a derivation. Manifolds with a Poisson algebra structure are known as Poisson manifolds, of which the symplectic manifolds are a special case. The Poisson bracket $\{\cdot, \cdot\}$ on a symplectic manifold $(M, \omega)$ is a bilinear operation on differentiable functions, defined by

$$
\{a, b\}=\omega\left(X_{a}, X_{b}\right)
$$

where $X_{a}, X_{b}$ are the Hamiltonian vector fields generated by the functions $a$ and $b$; the Poisson bracket of two functions on $M$ is itself a function on $M$. The Poisson bracket is antisymmetric because:

$$
\{a, b\}=\omega\left(X_{a}, X_{b}\right)=-\omega\left(X_{b}, X_{a}\right)=-\{b, a\} .
$$

Let $\mathbb{K}$ be a field. Recall that a Lie algebra $(\mathfrak{g},[\cdot, \cdot])$ over $\mathbb{K}$ consists of a vector space $\mathfrak{g}$ and a pairing $[\cdot, \cdot]: \mathfrak{g} \times \mathfrak{g} \rightarrow \mathfrak{g}$, called a Lie bracket, satisfying the relations of antisymmetry and the Jacobi identity

$$
[u,[v, w]]+[v,[w, u]]+[w,[u, v]]=0
$$

for all $u, v, w \in \mathfrak{g}$. Given two Lie algebras $(\mathfrak{g},[\cdot, \cdot])$ and $\left(\mathfrak{g}^{\prime},[\cdot, \cdot]^{\prime}\right)$, a morphism $\phi: \mathfrak{g} \rightarrow \mathfrak{g}^{\prime}$ of Lie algebras over $\mathbb{K}$ is a morphism of vector spaces which is compatible with the Lie bracket, i.e.

$$
\phi([u, v])=[\phi(u), \phi(v)]^{\prime} .
$$

Let $\mathcal{A}$ be an algebra over $\mathbb{K}$. Recall that a derivation of $\mathcal{A}$ (over $\mathbb{K}$ ) is a $\mathbb{K}$-linear $\operatorname{map} \delta: \mathcal{A} \rightarrow \mathcal{A}$ such that $\delta(a b)=(\delta(a)) b+a \delta(b)$. It is well known that the module $\operatorname{Der}(\mathcal{A})$ of derivations of $\mathcal{A}$, with bracket given by $[\alpha, \beta](a)=\alpha(\beta(a))-\beta(\alpha(a))$, where $\alpha, \beta \in \operatorname{Der} \mathcal{A}, a \in \mathcal{A}$, is a Lie algebra. If $\mathfrak{g}$ is a Lie algebra over $\mathbb{K}$, an action of $\mathfrak{g}$ on $\mathcal{A}$ is a Lie algebra homomorphism $\omega: \mathfrak{g} \rightarrow \operatorname{Der}(\mathcal{A})$. More details can be found in [11, 12, 24, 26].

A Poisson algebra is an associative algebra with a Lie bracket with the action of a derivation. Let us recall the definition: 
Definition 3.1. A Poisson algebra $(\mathcal{A},\{\cdot, \cdot\})$ is a pair consisting of an algebra $\mathcal{A}$ (over a field $\mathbb{K}$ ) together with a bilinear map $\{\cdot, \cdot\}: \mathcal{A} \times \mathcal{A} \rightarrow \mathcal{A}$ such that

(1) $\{a, b\}=-\{b, a\}$

(2) $\{\lambda a, \mu b\}=\lambda \mu\{a, b\}$

(3) $\{a b, c\}=a\{b, c\}+\{a, c\} b$

(4) $\{a,\{b, c\}\}+\{b,\{c, a\}\}+\{c,\{a, b\}\}=0$

for all $a, b, c \in \mathcal{A}$ and $\lambda, \mu \in \mathbb{K}$. The map $\{\cdot, \cdot\}$ is called the Poisson bracket.

Condition (4) guarantees that $\{\cdot, \cdot\}$ is a Lie bracket on $\mathcal{A}$, and condition (3) implies that for all $a \in \mathcal{A}$, the operator $\{a, \cdot\}$ is a derivation of the algebra $\mathcal{A}$.

Example 3.2. Let $\mathbb{C}[x, y, z]$ be the polynomial ring in three variables and write $x^{1}=x, x^{2}=y$ and $x^{3}=z$. For arbitrary $C \in \mathbb{C}[x, y, z]$, one can show that

$$
\left\{x^{i}, x^{j}\right\}=\epsilon^{i j k} \partial_{k} C,
$$

where $\epsilon^{i j k}$ denotes the totally antisymmetric symbol with $\epsilon^{123}=1$, is a Poisson bracket on $\mathbb{C}[x, y, z]$. Note that since

$$
\left\{x^{i}, C\right\}=\left\{x^{i}, x^{k}\right\} \partial_{k} C=\epsilon^{i k l}\left(\partial_{l} C\right)\left(\partial_{k} C\right)=0,
$$

due to the fact that $\epsilon^{i k l}=-\epsilon^{i l k}$ and $\left(\partial_{l} C\right)\left(\partial_{k} C\right)$ is symmetric when interchanging $k$ and $l$, the Poisson structure is well defined on the quotient algebra $\mathbb{C}[x, y, z] /(C)$. For instance, let us choose $C=\frac{1}{2}\left(x^{2}+y^{2}+z^{2}-1\right)$, giving

$$
\{x, y\}=\partial_{z} C=z,\{y, z\}=\partial_{x} C=x \quad \text { and } \quad\{z, x\}=\partial_{y} C=y
$$

and let us explicitly check (4)

$$
\begin{aligned}
\{\{x, y\}, z\}+\{\{y, z\}, x\}+\{\{z, x\}, y\} & =\left\{\partial_{z} C, z\right\}+\left\{\partial_{x} C, x\right\}+\left\{\partial_{y} C, y\right\} \\
& =\{z, z\}+\{x, x\}+\{y, y\}=0 .
\end{aligned}
$$

By the above argument this also defines a Poisson structure on the quotient algebra

$$
\mathcal{A}=\mathbb{C}[x, y, z] /\left(x^{2}+y^{2}+z^{2}-1\right) .
$$

Let us recall some basic facts of direct sums and tensor products of Poisson algebras. Firstly, we recall that the tensor product $\mathcal{A} \otimes \mathcal{A}^{\prime}$ of two Poisson algebras $(\mathcal{A},\{\cdot, \cdot\})$ and $\left(\mathcal{A}^{\prime},\{\cdot, \cdot\}^{\prime}\right)$ is a Poisson algebra with Poisson bracket

$$
\left\{a_{1} \otimes a_{2}, b_{1} \otimes b_{2}\right\}=\left\{a_{1}, b_{1}\right\} \otimes a_{2} b_{2}+a_{1} b_{1} \otimes\left\{a_{2}, b_{2}\right\}^{\prime},
$$

for $a_{1}, b_{1} \in \mathcal{A}$ and $a_{2}, b_{2} \in \mathcal{A}^{\prime}$ (see e.g. [9]).

Secondly, the direct sum of two Poisson algebras $\mathcal{A} \oplus \mathcal{A}^{\prime}$ is a Poisson algebra with Poisson bracket

$$
\left\{\left(a_{1}, a_{2}\right),\left(b_{1}, b_{2}\right)\right\}=\left(\left\{a_{1}, b_{1}\right\},\left\{a_{2}, b_{2}\right\}^{\prime}\right)
$$

for $a_{1}, b_{1} \in \mathcal{A}$ and $a_{2}, b_{2} \in \mathcal{A}^{\prime}$. 


\subsection{Kähler-Poisson algebras}

Let us consider a unital Poisson algebra $(\mathcal{A},\{\cdot, \cdot\})$, over a field $\mathbb{K}$ (either $\mathbb{R}$ or $\mathbb{C}$ ) and let $\left\{x^{1}, \ldots, x^{m}\right\}$ be a set of distinguished elements of $\mathcal{A}$. These elements play the role of functions providing an embedding into $\mathbb{R}^{m}$ in the geometrical case [1, 2].

Kähler-Poisson algebras as defined below are, in a natural way, metric LieRinehart algebras, which implies that the results of Chapter 2 can be applied; in particular, there exists a unique torsion-free metric connection on every KählerPoisson algebra. Lie-Rinehart algebras related to Poisson algebras have been extensively studied by Huebschmann [12, 13, although metric aspects were not considered there. In the following, we study metric aspects by defining Kähler-Poisson algebras as algebraic analogues of the algebra of functions on Kähler manifolds. At the end, detailed examples are provided in order to illustrate the novel concepts. We continue with the main definition of this thesis.

Definition 3.3. Let $(\mathcal{A},\{\cdot, \cdot\})$ be a Poisson algebra over $\mathbb{K}$ and let $x^{1}, \ldots, x^{m} \in \mathcal{A}$. Given a symmetric $m \times m$ matrix $g=\left(g_{i j}\right)$ with entries $g_{i j} \in \mathcal{A}$, for $i, j=1, \ldots, m$, we say that the triple $\mathcal{K}=\left(\mathcal{A}, g,\left\{x^{1}, \ldots, x^{m}\right\}\right)$ is a Kähler-Poisson algebra if there exists $\eta \in \mathcal{A}$ such that

$$
\sum_{i, j, k, l}^{m} \eta\left\{a, x^{i}\right\} g_{i j}\left\{x^{j}, x^{k}\right\} g_{k l}\left\{x^{l}, b\right\}=-\{a, b\}
$$

for all $a, b \in \mathcal{A}$. Moreover, we set $\mathcal{P}^{i j}=\left\{x^{i}, x^{j}\right\}$.

Remark 3.4. From now on, we shall use the differential geometric convention that repeated indices are summed over from 1 to $m$, and omit explicit summation symbols.

Remark 3.5. If $\mathcal{A}$ is generated by $x^{1}, \ldots, x^{m}$ (that is, every element of $\mathcal{A}$ is a polynomial in the variables $x^{1}, \ldots, x^{m}$ ) then the Poisson structure is completely determined by $\mathcal{P}^{i j}$.

Note that if $a$ and $b$ are polynomials in $x^{1}, \ldots, x^{m}$ then

$$
\{a, b\}=\left\{x^{i}, x^{j}\right\} \frac{\partial a}{\partial x^{i}} \frac{\partial b}{\partial x^{j}}=\mathcal{P}^{i j} \frac{\partial a}{\partial x^{i}} \frac{\partial b}{\partial x^{j}}
$$

due to the fact that the Poisson bracket is a derivation in both arguments see Definition 3.1(3). For example in the case of two generators, where the Poisson structure is determined by $\{x, y\}$,

$$
\left\{x^{2}+x, y\right\}=2 x\{x, y\}+\{x, y\} .
$$

For an algebra generated by $x^{1}, \ldots, x^{m}$, condition 3.3 is equivalent to

$$
\eta \mathcal{P} g \mathcal{P} g \mathcal{P}=-\mathcal{P}
$$

where $\mathcal{P}=\left(\mathcal{P}^{i j}\right)$ and $g=\left(g_{i j}\right)$ (in Definition 3.3) are considered as $(m \times m)$ matrices and the product in the expression above is matrix multiplication. 
In Section 3.6 we will provide a rather general way to associate a localization $\mathcal{A}\left[\alpha^{-1}\right]$ and a metric $g$ to a Poisson algebra $(\mathcal{A},\{\cdot, \cdot\})$, such that

$$
\left(\mathcal{A}\left[\alpha^{-1}\right], g,\left\{x^{1}, \ldots, x^{m}\right\}\right)
$$

is a Kähler-Poisson algebra with $\eta=\alpha^{-1}$. Namely, as we shall see, finding a metric $g$ and $\alpha \in \mathcal{A}$ such that

$$
\mathcal{P} g \mathcal{P} g \mathcal{P}=-\alpha \mathcal{P}
$$

may be easy; if $\alpha$ is invertible then one obtains a Kähler-Poisson algebra with $\eta=\alpha^{-1}$. However, if $\alpha$ is not invertible, then one may add the inverse to the algebra and obtain a localization which defines a Kähler-Poisson algebra with $\eta=\alpha^{-1}$. Let us now consider a few examples of Kähler-Poisson algebras. More details can be found in [4].

Example 3.6. Let $\mathcal{A}=\mathbb{C}[x, y, z]$ and define a Poisson algebra structure by setting

$$
\{x, y\}=\lambda_{1} \quad\{y, z\}=\lambda_{2} \quad\{z, x\}=\lambda_{3}
$$

with $\lambda_{1}, \lambda_{2}, \lambda_{3} \in \mathbb{C}$ (note that the Jacobi identity is trivially satisfied for a constant Poisson bracket). One sets

$$
\mathcal{P}=\left(\mathcal{P}^{i j}\right)=\left(\begin{array}{ccc}
0 & \lambda_{1} & -\lambda_{3} \\
-\lambda_{1} & 0 & \lambda_{2} \\
\lambda_{3} & -\lambda_{2} & 0
\end{array}\right)
$$

and it is easy to check that for the matrix

$$
g=\mu\left(\begin{array}{lll}
1 & 0 & 0 \\
0 & 1 & 0 \\
0 & 0 & 1
\end{array}\right)
$$

with $\mu \in \mathbb{C}$, one obtains

$$
\mathcal{P} g \mathcal{P} g \mathcal{P}=-\mu\left(\lambda_{1}^{2}+\lambda_{2}^{2}+\lambda_{3}^{2}\right) \mathcal{P}
$$

Hence, $(\mathcal{A}, g,\{x, y, z\})$ is a Kähler-Poisson algebra with

$$
\eta=\frac{1}{\mu\left(\lambda_{1}^{2}+\lambda_{2}^{2}+\lambda_{3}^{2}\right)} \in \mathbb{C} .
$$

Example 3.7. As explained in Example 3.2. $\mathcal{A}=\mathbb{C}[x, y, z] /\left(x^{2}+y^{2}+z^{2}-1\right)$ is a Poisson algebra with respect to $\{x, y\}=z,\{y, z\}=x$ and $\{z, x\}=y$. With

$$
\mathcal{P}=\left(\mathcal{P}^{i j}\right)=\left(\begin{array}{ccc}
0 & z & x \\
-z & 0 & y \\
-x & -y & 0
\end{array}\right)
$$

and

$$
g=\left(\begin{array}{lll}
1 & 0 & 0 \\
0 & 1 & 0 \\
0 & 0 & 1
\end{array}\right)
$$


one obtains

$$
\mathcal{P} g \mathcal{P} g \mathcal{P}=-\mathcal{P}
$$

Therefore, $(\mathcal{A}, g,\{x, y, z\})$ is a Kähler-Poisson algebra with $\eta=1$.

Example 3.8. Let $\mathcal{A}$ be a Poisson algebra generated by two elements $x^{1}=x$ and $x^{2}=y$. Let

$$
\mathcal{P}=\left(\mathcal{P}^{i j}\right)=\left(\begin{array}{cc}
0 & \{x, y\} \\
-\{x, y\} & 0
\end{array}\right)
$$

It is easy to check that for the matrix

$$
g=\left(\begin{array}{cc}
x & x+y \\
x+y & y
\end{array}\right), \text { with } \quad \operatorname{det}(g)=x y-(x+y)^{2}
$$

we obtain,

$$
\mathcal{P} g \mathcal{P} g \mathcal{P}=-\{x, y\}^{2} \operatorname{det}(g) \mathcal{P}
$$

implying that $\eta=\left(\{x, y\}^{2} \operatorname{det}(g)\right)^{-1}$. However, if this inverse does not exist in the algebra, one can localize $\mathcal{A}$, as long as $\{x, y\}^{2} \operatorname{det}(g)$ is not a zero-divisor, to obtain a Kähler-Poisson algebra

$$
\mathcal{K}=\left(\mathcal{A}\left[\left(\{x, y\}^{2} \operatorname{det}(g)\right)^{-1}\right], g,\{x, y\}\right) .
$$

Example 3.9. Let $\mathcal{A}$ be a Poisson algebra generated by one element $x$. Let $x^{1}=x$, $x^{2}=2 x$ giving

$$
\mathcal{P}=\left(\left\{x^{i}, x^{j}\right\}\right)=\left(\begin{array}{cc}
0 & \{x, 2 x\} \\
-\{x, 2 x\} & 0
\end{array}\right)=\left(\begin{array}{ll}
0 & 0 \\
0 & 0
\end{array}\right),
$$

and therefore $\mathcal{P} g \mathcal{P} g \mathcal{P}=0$ for any metric $g$. Hence, for arbitrary $g$ and $\eta$ it holds that $\eta \mathcal{P} g \mathcal{P} g \mathcal{P}=-\mathcal{P}$ implying that $(\mathcal{A}, g,\{x, 2 x\})$ is a Kähler-Poisson algebra.

Example 3.10. Let $\mathcal{A}$ be a Poisson algebra generated by two elements $x$ and, $y$. Let $x^{1}=x+y, x^{2}=x-y$ and

$$
\mathcal{P}=\left(\begin{array}{cc}
0 & \{x+y, x-y\} \\
-\{x+y, x-y\} & 0
\end{array}\right)=\left(\begin{array}{cc}
0 & -2\{x, y\} \\
2\{x, y\} & 0
\end{array}\right)
$$

It is easy to check that for the matrix

$$
g=\left(\begin{array}{cc}
\{x, y\} & 1 \\
1 & \{x, y\}
\end{array}\right), \text { where } \operatorname{det}(g)=\{x, y\}^{2}-1
$$

one obtains,

$$
\mathcal{P} g \mathcal{P} g \mathcal{P}=-\{x+y, x-y\}^{2}\left(\{x, y\}^{2}-1\right) \mathcal{P}
$$


and

$$
\begin{aligned}
\eta=\left(\{x+y, x-y\}^{2} \operatorname{det}(g)\right)^{-1} & =\left(\{x+y, x-y\}^{2}\left(\{x, y\}^{2}-1\right)\right)^{-1} \\
& =\left((-2\{x, y\})^{2}\left(\{x, y\}^{2}-1\right)\right)^{-1}
\end{aligned}
$$

Thus, as long as $\{x+y, x-y\}^{2} \operatorname{det}(g)$ is not a zero-divisor, one can localize to obtain a Kähler-Poisson algebra

$$
\mathcal{K}=\left(\mathcal{A}\left[\left(4\{x, y\}^{2} \operatorname{det}(g)\right)^{-1}\right], g,\{x, y\}\right) .
$$

Given a Kähler-Poisson algebra $\mathcal{K}=\left(\mathcal{A}, g,\left\{x^{1}, \ldots, x^{m}\right\}\right)$, we let $\mathfrak{g}$ denote the $\mathcal{A}$-module generated by all inner derivations, i.e.

$$
\mathfrak{g}=\left\{a_{1}\left\{c^{1}, .\right\}+\ldots+a_{N}\left\{c^{N}, .\right\}: a_{i}, c^{i} \in \mathcal{A} \quad \text { and } \quad N \in \mathbb{N}\right\}
$$

It is standard fact that $\mathfrak{g}$ is a Lie algebra over $\mathbb{K}$ with respect to the bracket

$$
[\alpha, \beta](a)=\alpha(\beta(a))-\beta(\alpha(a))
$$

where $\alpha, \beta \in \mathfrak{g}$ and $a \in \mathcal{A}$ (see e.g. [10]).

Let us now show that $(\mathcal{A}, \mathfrak{g})$ is a Lie-Rinehart algebra (cf. also Example 2.2). Indeed, choosing $\omega(\alpha)=\alpha$ for all $\alpha \in \mathfrak{g}$, let show that the conditions in Definition 2.1 are satisfied. The first condition

$$
\omega(a \alpha)(b)=a(\omega(\alpha)(b)
$$

is satisfied, since $\omega(a \alpha)(b)=a \alpha(b)$ and $a(\omega(\alpha)(b)=a \alpha(b)$, where $a, b \in \mathcal{A}$. The second condition

$$
[\alpha, a \beta](c)=a[\alpha, \beta](c)+(\omega(\alpha)(a) \beta(c)
$$

is also satisfied, since

$$
[\alpha, a \beta](c)=\alpha(a \beta(c))-a \beta(\alpha(c))=a \alpha \beta(c)+\alpha(a) \beta(c)-a \beta(\alpha(c))
$$

and

$$
\begin{aligned}
a[\alpha, \beta](c)+(\omega(\alpha)(a) \beta(c) & =a(\alpha(\beta(c))-\beta(\alpha(c)))+(\alpha(a)) \beta(c) \\
& =a \alpha \beta(c)-a \beta(\alpha(c))+\beta(c) \alpha(a)
\end{aligned}
$$

for $a, c \in \mathcal{A}$. Hence, $(\mathcal{A}, \mathfrak{g})$ is a Lie-Rinehart algebra.

The matrix $g$ provides a metric on $\mathfrak{g}$, defined by

$$
g(\alpha, \beta)=\alpha\left(x^{i}\right) g_{i j} \beta\left(x^{i}\right) .
$$

To the metric $g$ one can associate a map $\hat{g}: \mathfrak{g} \rightarrow \mathfrak{g}^{*}$ defined as

$$
\hat{g}(\alpha)(\beta)=g(\alpha, \beta) .
$$

The following result shows that $g$ is a metric on $\mathfrak{g}$ in the sense of Definition 2.4 
Proposition 3.11 ([3]). If $\mathcal{K}=\left(\mathcal{A}, g,\left\{x^{1}, \ldots, x^{m}\right\}\right)$ is a Kähler-Poisson algebra then the metric $g$ is non-degenerate; i.e. the map $\hat{g}: \mathfrak{g} \rightarrow \mathfrak{g}^{*}$ is a module isomorphism.

Therefore, Proposition 3.11 implies that $(\mathcal{A}, \mathfrak{g}, g)$ is a metric Lie-Rinehart algebra. Let us now introduce some notation for Kähler-Poisson algebras. We recall

$$
\mathcal{P}^{i j}=\left\{x^{i}, x^{j}\right\}
$$

and we set

$$
\mathcal{P}^{i}(a)=\left\{x^{i}, a\right\}
$$

for $a \in \mathcal{A}$, as well as

$$
\begin{aligned}
\mathcal{D}^{i j} & =\eta\left\{x^{i}, x^{l}\right\} g_{l k}\left\{x^{j}, x^{k}\right\} \\
\mathcal{D}^{i}(a) & =\eta\left\{x^{k}, a\right\} g_{k l}\left\{x^{l}, x^{i}\right\} .
\end{aligned}
$$

Note that $\mathcal{D}^{i j}=\mathcal{D}^{j i}$. The metric $g$ will be used to lower indices in analogy with differential geometry; e.g.

$$
\mathcal{P}^{i}{ }_{j}=\mathcal{P}^{i k} g_{k j} \quad \mathcal{D}^{i}{ }_{j}=\mathcal{D}^{i k} g_{k j} \quad \mathcal{D}_{i}=g_{i j} \mathcal{D}^{j} .
$$

With respect to this notation, $\sqrt{3.3}$ can be stated as

$$
\mathcal{D}_{i}(a) \mathcal{P}^{i}(b)=\{a, b\},
$$

and one immediately derives the following identities

$$
\mathcal{D}^{i j} \mathcal{P}_{j}(a)=\mathcal{P}^{i}(a) \quad \mathcal{P}^{i j} \mathcal{D}_{j}(a)=\mathcal{P}^{i}(a) \quad \mathcal{D}^{i}{ }_{j} \mathcal{D}^{j k}=\mathcal{D}^{j k} .
$$

Furthermore, there is a natural embedding $\iota: \mathfrak{g} \rightarrow \mathcal{A}^{m}$, given by

$$
\iota\left(a_{i}\left\{b^{i}, .\right\}\right)=a_{i}\left\{b^{i}, x^{k}\right\} e_{k},
$$

where $\mathcal{A}^{m}$ is a free module and $\left\{e_{k}\right\}_{k=1}^{m}$ denotes the canonical basis of $\mathcal{A}^{m}$, and $g$ induces a bilinear form on $\mathcal{A}^{m}$ via

$$
g(X, Y)=X^{i} g_{i j} Y^{j}
$$

for $X=X^{i} e_{i} \in \mathcal{A}^{m}$ and $Y=Y^{i} e_{i} \in \mathcal{A}^{m}$. Finally, we introduce $\mathcal{D}: \mathcal{A}^{m} \rightarrow \mathcal{A}^{m}$ by setting

$$
\mathcal{D}(X)=\mathcal{D}^{i}{ }_{j} X^{j} e_{i}
$$

for $X=X^{i} e_{i} \in \mathcal{A}^{m}$.

Proposition $3.12([3])$. The map $\mathcal{D}: \mathcal{A}^{m} \rightarrow \mathcal{A}^{m}$ is an orthogonal projection, i.e.

$$
\mathcal{D}^{2}(X)=\mathcal{D}(X) \quad \text { and } \quad g(\mathcal{D}(X), Y)=g(X, \mathcal{D}(Y))
$$

for all $X, Y \in \mathcal{A}^{m}$. 
From Proposition 3.12 we conclude that $T \mathcal{A}=i m(\mathcal{D})$ is a finitely generated projective module. Moreover, one can show that the map $\iota: \mathfrak{g} \rightarrow \mathcal{A}^{m}$ is in fact an isomorphism from $\mathfrak{g}$ to $T \mathcal{A}$, leading to the following result.

Proposition 3.13. $\mathfrak{g}$ is a finitely generated projective module and the $\mathcal{A}$-module $\mathfrak{g}$ is generated by $\left\{\mathcal{D}^{1}, \ldots, \mathcal{D}^{m}\right\}$.

Note that the above result is clearly not dependent on whether or not the underlying Poisson algebra has the structure of a Kähler-Poisson algebra, as the definition of $\mathfrak{g}$ involves only inner derivations. Hence, as soon as the Poisson algebra admits the structure of a Kähler-Poisson algebra, it follows that the module of inner derivations is projective.

We will now give a generalization of Examples 3.8 and 3.10 . As shown below, every Poisson-algebra generated by two and three elements is associated with a Kähler-Poisson algebra. Let $\mathcal{A}$ be a Poisson algebra generated by two elements $x^{1}=x \in \mathcal{A}$ and, $x^{2}=y \in \mathcal{A}$ and set

$$
\mathcal{P}=\left(\begin{array}{cc}
0 & \{x, y\} \\
-\{x, y\} & 0
\end{array}\right)
$$

It is easy to check that for an arbitrary symmetric matrix

$$
g=\left(\begin{array}{ll}
g_{11} & g_{12} \\
g_{12} & g_{22}
\end{array}\right),
$$

with $g_{11}, g_{12}, g_{22} \in \mathcal{A}$, one obtains

$$
\mathcal{P} g \mathcal{P} g \mathcal{P}=-\{x, y\}^{2} \operatorname{det}(g) \mathcal{P}
$$

giving $\eta=\left(\{x, y\}^{2} \operatorname{det}(g)\right)^{-1}$. Thus, as long as $\{x, y\}^{2} \operatorname{det}(g)$ is not a zero-divisor, one can localize $\mathcal{A}$ to obtain a Kähler-Poisson algebra

$$
\mathcal{K}=\left(\mathcal{A}\left[\left(\{x, y\}^{2} \operatorname{det}(g)\right)^{-1}\right], g,\{x, y\}\right) .
$$

Similarly, let $\mathcal{A}$ be a Poisson algebra generated by three elements

$$
x^{1}=x \quad x^{2}=y \quad x^{3}=z .
$$

Writing $\{x, y\}=a,\{y, z\}=b$ and $\{z, x\}=c$, i.e.

$$
\mathcal{P}=\left(\begin{array}{ccc}
0 & a & -c \\
-a & 0 & b \\
c & -b & 0
\end{array}\right)
$$

It is easy to check that for an arbitrary symmetric matrix $g$

$$
\mathcal{P} g \mathcal{P} g \mathcal{P}=-\tau \mathcal{P}
$$

with

$$
\tau=a^{2}|g|_{33}+b^{2}|g|_{11}+c^{2}|g|_{22}+2 a b|g|_{31}-2 a c|g|_{32}-2 b c|g|_{21}
$$


and $|g|_{i j}$ denotes the determinant of the matrix obtained from $g$ by deleting the $i$ 'th row and the $j$ 'th column. Thus, via localization, one can construct the KählerPoisson algebra

$$
\mathcal{K}=\left(\mathcal{A}\left[\tau^{-1}\right], g,\{x, y, z\}\right) .
$$

In particular, if $g=\operatorname{diag}(\lambda, \lambda, \lambda)$, then $\tau=\lambda^{2}\left(a^{2}+b^{2}+c^{2}\right)$.

Example 3.14. Let $\mathcal{A}_{C}=\mathbb{C}[x, y, z]$ and define a Poisson structure on $\mathcal{A}_{C}$ via (cf. Example 3.2)

$$
\{x, y\}=\partial_{z} C \quad\{y, z\}=\partial_{x} C \quad\{z, x\}=\partial_{y} C
$$

i.e

$$
\mathcal{P}=\left(\begin{array}{ccc}
0 & \partial_{z} C & -\partial_{y} C \\
-\partial_{z} C & 0 & \partial_{x} C \\
\partial_{y} C & -\partial_{x} C & 0
\end{array}\right)
$$

Consider the Kähler-Poisson algebra obtained from $\mathcal{A}_{C}$ by choosing the metric $g=\operatorname{diag}(\mu, \mu, \mu)$. One obtains

$$
\mathcal{P} g \mathcal{P} g \mathcal{P}=-\mu^{2}\left(\left(\partial_{z} C\right)^{2}+\left(\partial_{y} C\right)^{2}+\left(\partial_{x} C\right)^{2}\right) \mathcal{P}
$$

giving

$$
\eta=\mu^{2}\left(\left(\partial_{x} C\right)^{2}+\left(\partial_{y} C\right)^{2}+\left(\partial_{z} C\right)^{2}\right)^{-1}
$$

For instance, for

$$
C=\frac{1}{n+1}\left(x^{n+1}+y^{n+1}+z^{n+1}\right)-\lambda x y z,
$$

with $\lambda \in \mathbb{R}$, one obtains

$$
\mathcal{P} g \mathcal{P} g \mathcal{P}=-\mu^{2}\left(\left(x^{n}-\lambda y z\right)^{2}+\left(y^{n}-\lambda x z\right)^{2}+\left(z^{n}-\lambda x y\right)^{2}\right) \mathcal{P} .
$$

Therefore,

$$
\eta=\left(\mu^{2}\left(\left(x^{n}-\lambda y z\right)^{2}+\left(y^{n}-\lambda x z\right)^{2}+\left(z^{n}-\lambda x y\right)^{2}\right)\right)^{-1},
$$

giving the Kähler-Poisson algebra

$$
\mathcal{K}=\left(\mathcal{A}\left[\left(\mu^{2}\left(\left(x^{n}-\lambda y z\right)^{2}+\left(y^{n}-\lambda x z\right)^{2}+\left(z^{n}-\lambda x y\right)^{2}\right)\right)^{-1}\right], g,\{x, y, z\}\right) .
$$

\subsection{Homomorphisms of Kähler-Poisson algebras}

We are interested in understanding maps between Kähler-Poisson algebras as well as their algebraic properties. Let $\mathcal{K}$ and $\mathcal{K}^{\prime}$ be Kähler-Poisson algebras. We would like to understand when $\mathcal{K}$ and $\mathcal{K}^{\prime}$ are isomorphic. As Kähler-Poisson algebras are 
also metric Lie-Rinehart algebras, as we saw in Chapter 3 , we shall require that a morphism of Kähler-Poisson algebras is also a morphism of metric Lie-Rinehart algebras. However, as the definition of a Kähler-Poisson algebra involves the choice of a set of distinguished elements, we require a morphism to respect the subalgebra generated by these elements. Furthermore, we will introduce subalgebras, direct sums and tensor products of Kähler-Poisson algebras by using the concept of morphisms. To this end, we start by making the following definition.

Definition 3.15. Given a Kähler-Poisson algebra $\mathcal{K}=\left(\mathcal{A}, g,\left\{x^{1}, \ldots, x^{m}\right\}\right)$, let $\mathcal{A}_{\text {fin }} \subseteq \mathcal{A}$ denote the subalgebra generated by $\left\{x^{1}, \ldots, x^{m}\right\}$.

Note that $\mathcal{A}_{\text {fin }}$ is not necessarily a Poisson subalgebra of $\mathcal{A}$. Equipped with this definition, we introduce morphisms of Kähler-Poisson algebras in the following way.

Definition 3.16. Let $\mathcal{K}=\left(\mathcal{A}, g,\left\{x^{1}, \ldots, x^{m}\right\}\right)$ and $\mathcal{K}^{\prime}=\left(\mathcal{A}^{\prime}, g^{\prime},\left\{y^{1}, \ldots, y^{m \prime}\right\}\right)$ be Kähler-Poisson algebras together with their corresponding modules of derivations $\mathfrak{g}$ and $\mathfrak{g}^{\prime}$, respectively. A morphism of Kähler-Poisson algebras is a pair of maps $(\phi, \psi)$, with $\phi: \mathcal{A} \rightarrow \mathcal{A}^{\prime}$ a Poisson algebra homomorphism and $\psi: \mathfrak{g} \rightarrow \mathfrak{g}^{\prime}$ a Lie algebra homomorphism, such that

1. $\psi(a \alpha)=\phi(a) \psi(\alpha)$,

2. $\phi(\alpha(a))=\psi(\alpha)(\phi(a))$,

3. $\phi(g(\alpha, \beta))=g^{\prime}(\psi(\alpha), \psi(\beta))$,

4. $\phi\left(\mathcal{A}_{\text {fin }}\right) \subseteq \mathcal{A}_{\text {fin }}^{\prime}$,

for all $a \in \mathcal{A}$ and $\alpha, \beta \in \mathfrak{g}$.

Remark 3.17. Note that a morphism of Kähler-Poisson algebras is also a morphism of the underlying Lie-Rinehart algebras.

Furthermore, in next Proposition we show that the composition of two KählerPoisson algebra morphisms is a again morphism of Kähler-Poisson algebras.

Proposition 3.18. Let $\mathcal{K}=\left(\mathcal{A}, g,\left\{x^{1}, \ldots, x^{m}\right\}\right), \mathcal{K}^{\prime}=\left(\mathcal{A}^{\prime}, g^{\prime},\left\{y^{1}, \ldots, y^{m^{\prime}}\right\}\right)$ and $\mathcal{K}^{\prime \prime}=\left(\mathcal{A}^{\prime \prime}, g^{\prime \prime},\left\{z^{1}, \ldots, z^{m \prime \prime}\right\}\right)$ be a Kähler-Poisson algebras. If $(\phi, \psi): \mathcal{K} \rightarrow \mathcal{K}^{\prime}$ and $\left(\phi^{\prime}, \psi^{\prime}\right): \mathcal{K}^{\prime} \rightarrow \mathcal{K}^{\prime \prime}$ are homomorphisms of Kähler-Poisson algebras then the composition $\left(\phi^{\prime} \circ \phi, \psi^{\prime} \circ \psi\right): \mathcal{K} \rightarrow \mathcal{K}^{\prime \prime}$ is a homomorphism of Kähler-Poisson algebras.

Remark 3.19. Note that, the composition of Kähler-Poisson algebra morphism is associative and the identity $\left(\operatorname{id}_{\mathcal{A}}, \operatorname{id}_{\mathfrak{g}}\right)$ of $\mathcal{K}=\left(\mathcal{A}, g,\left\{x^{1}, \ldots, x^{m}\right\}\right)$ with id $\mathcal{A}_{\mathcal{A}}: \mathcal{A} \rightarrow \mathcal{A}$ and $\operatorname{id}_{\mathfrak{g}}: \mathfrak{g} \rightarrow \mathfrak{g}$ is a morphism of Kähler-Poisson algebras.

Remark 3.20. An isomorphism of Kähler-Poisson algebras is a morphism $(\phi, \psi)$ of Kähler-Poisson algebras such that $\phi$ is a Poisson algebra isomorphism and $\phi\left(\mathcal{A}_{\text {fin }}\right)=\mathcal{A}_{\text {fin }}^{\prime}$. Observe that, in this case, $\psi$ can always be constructed from $\phi$ (via 2 in Definition 3.16). When two Kähler-Poisson algebras $\mathcal{K}$ and $\mathcal{K}^{\prime}$ are isomorphic, we write $\mathcal{K} \cong \mathcal{K}^{\prime}$. 
Before continuing, we need to introduce some notation. Let $(\mathcal{A},\{\cdot, \cdot\})$ and $\left(\mathcal{A}^{\prime},\{\cdot, \cdot\}^{\prime}\right)$ be a Poisson algebras and let $x^{i} \in \mathcal{A}$ for $i=1, \ldots, m$. If $p \in \mathcal{A}$ is a polynomial in $\left\{x^{1}, \ldots, x^{m}\right\}$ then, using Leibniz rule, one may compute

$$
\{p, a\}=\frac{\partial p}{\partial x^{i}}\left\{x^{i}, a\right\}
$$

where $\frac{\partial p}{\partial x^{i}}$ denotes the formal derivative of the polynomial $p$ with respect to the variable $x^{i}$. Note that, in general, $\frac{\partial p}{\partial x^{i}}$ is itself not well-defined in the algebra, since there might exist several different (but equivalent) representations of $p$ as a polynomial in $x^{1}, \ldots, x^{m}$, and the formal derivative then yields several, possibly non-equivalent, elements of the algebra. However, the combination in $\sqrt{3.8}$ is always well-defined, and gives the same result for all representations of $p$.

Given a matrix $M=\left(m_{i j}\right)$ over $\mathcal{A}$, we set $\phi(M)=\left(\phi\left(m_{i j}\right)\right)$. Given a morphism $(\phi, \psi):\left(\mathcal{A}, g,\left\{x^{1}, \ldots, x^{m}\right\}\right) \rightarrow\left(\mathcal{A}^{\prime}, g^{\prime},\left\{y^{1}, \ldots, y^{m^{\prime}}\right\}\right)$, it will be convenient to introduce the notation

$$
A_{\alpha}^{i}=\frac{\partial \phi\left(x^{i}\right)}{\partial y^{\alpha}}
$$

(keeping in mind that this is not well-defined by itself); recall that if $(\phi, \psi)$ is a morphism of Kähler-Poisson algebras, then $\phi\left(\mathcal{A}_{\text {fin }}\right) \subseteq \mathcal{A}_{\text {fin }}^{\prime}$, ensuring that $\phi\left(x^{i}\right)$ is indeed a polynomial in $y^{1}, \ldots, y^{m^{\prime}}$. This notation allows us to write

$$
\phi\left(\left\{x^{i}, x^{j}\right\}\right)=\left\{\phi\left(x^{i}\right), \phi\left(x^{j}\right)\right\}^{\prime}=A_{\alpha}^{i}\left\{y^{\alpha}, y^{\beta}\right\}^{\prime} A^{j}{ }_{\beta}
$$

in matrix notation as

$$
\phi(\mathcal{P})=A \mathcal{P}^{\prime} A^{T}
$$

where $\mathcal{P}=\left(\left\{x^{i}, x^{j}\right\}\right)$ and $\mathcal{P}^{\prime}=\left(\left\{y^{\alpha}, y^{\beta}\right\}^{\prime}\right)$.

In the following, we shall consider a number of examples in order to explore when Kähler-Poisson algebras are isomorphic. A standing assumption is that all the algebras have been properly localized to allow for the construction of a KählerPoisson algebra (cf. examples in Chapter 3). Now given two Kähler-Poisson algebras $\mathcal{K}=\left(\mathcal{A}, g,\left\{x^{1}, \ldots, x^{m}\right\}\right)$ and $\mathcal{K}^{\prime}=\left(\mathcal{A}^{\prime}, g^{\prime},\left\{y^{1}, \ldots, y^{m^{\prime}}\right\}\right)$, we would like to understand when they are isomorphic. Let us start with the case when $\mathcal{A}=\mathcal{A}^{\prime}$, but where we choose two distinct sets of generators.

Example 3.21. Let $(\mathcal{A},\{\cdot, \cdot\})$ be a Poisson algebra generated by two elements $x, y$. From the previous general considerations we know that $\mathcal{K}=\left(\mathcal{A}, g,\left\{x^{1}, x^{2}\right\}\right)=$ $(\mathcal{A}, g,\{x, y\})$ is a Kähler-Poisson algebra for arbitrary symmetric matrices

$$
g=\left(\begin{array}{ll}
g_{11} & g_{12} \\
g_{12} & g_{22}
\end{array}\right),
$$

with

$$
\eta=\left(\{x, y\}^{2} \operatorname{det}(g)\right)^{-1}
$$


Now, let $\mathcal{K}^{\prime}=\left(\mathcal{A}, h,\left\{y^{1}, y^{2}, y^{3}\right\}\right)=(\mathcal{A}, h,\{x, y, x\})$ with

$$
\mathcal{P}^{\prime}=\left(\begin{array}{ccc}
0 & \lambda & 0 \\
-\lambda & 0 & -\lambda \\
0 & \lambda & 0
\end{array}\right)
$$

where $\lambda=\{x, y\}$. It is easy to check that for the symmetric matrix $h$

$$
h=\left(\begin{array}{ccc}
\frac{1}{4} g_{11} & \frac{1}{2} g_{12} & \frac{1}{4} g_{11} \\
\frac{1}{2} g_{12} & g_{22} & \frac{1}{2} g_{12} \\
\frac{1}{4} g_{11} & \frac{1}{2} g_{12} & \frac{1}{4} g_{11}
\end{array}\right),
$$

one obtains

$$
\mathcal{P}^{\prime} h \mathcal{P}^{\prime} h \mathcal{P}^{\prime}=-\{x, y\}^{2} \operatorname{det}(g) \mathcal{P}^{\prime},
$$

giving $\eta^{\prime}=\left(\{x, y\}^{2} \operatorname{det}(g)\right)^{-1}=\eta$. Now let us check that $\mathcal{K} \cong \mathcal{K}^{\prime}$ by using Definition 3.16. From

$$
\mathfrak{g}=\left\{a_{1}\{x, \cdot\}+a_{2}\{y, \cdot\}: a_{1}, a_{2} \in \mathcal{A}\right\}
$$

and

$$
\begin{aligned}
\mathfrak{g}^{\prime} & =\left\{a_{1}\{x, \cdot\}+a_{2}\{y, \cdot\}+a_{3}\{x, \cdot\}: a_{1}, a_{2}, a_{3} \in \mathcal{A}\right\} \\
& =\left\{\left(a_{1}+a_{3}\right)\{x, \cdot\}+a_{2}\{y, \cdot\}: a_{1}, a_{2}, a_{3} \in \mathcal{A}\right\}
\end{aligned}
$$

we conclude that $\mathfrak{g}=\mathfrak{g}^{\prime}$. To prove that $\mathcal{K} \cong \mathcal{K}^{\prime}$, we need to define the maps $\phi: \mathcal{A} \rightarrow \mathcal{A}$ and $\psi: \mathfrak{g} \rightarrow \mathfrak{g}$. By choosing $\phi=i d$ and $\psi=i d$ we will show that $(\phi, \psi)$ is an isomorphism of Kähler Poisson algebras. Properties (1),(2),(4) in Definition 3.16 are easily seen to be satisfied. To check property (3), one needs to show that

$$
\phi(g(\alpha, \beta))=h(\psi(\alpha), \psi(\beta))=h(\alpha, \beta) .
$$

Starting from the right hand side we get

$$
\begin{aligned}
& h(\psi(\alpha), \psi(\beta))=h(\alpha, \beta)=\sum_{i, j=1}^{3} \alpha\left(y^{i}\right) h_{i j} \beta\left(y^{j}\right) \\
& =\alpha(x) g_{11} \beta(x)+\alpha(x) g_{12} \beta(y)+\alpha(y) g_{12} \beta(x)+\alpha(y) g_{22} \beta(y) .
\end{aligned}
$$

From the left hand side we get

$$
\begin{aligned}
\phi(g(\alpha, \beta)) & =\sum_{i, j=1}^{2} \alpha\left(x^{i}\right) g_{i j} \beta\left(x^{j}\right) \\
& =\alpha(x) g_{11} \beta(x)+\alpha(x) g_{12} \beta(y)+\alpha(y) g_{12} \beta(x)+\alpha(y) g_{22} \beta(y) .
\end{aligned}
$$

Therefore, $\phi(g(\alpha, \beta))=h(\psi(\alpha), \psi(\beta))$, which shows that $\mathcal{K} \cong \mathcal{K}^{\prime}$. 
Note that the above example extends to the case where we choose more (dependent) generators of the finitely generated algebra, giving many possible presentations of the same Kähler-Poisson algebra. Next, let us explore the case when we choose different, but the same number of, generators for the algebra.

Example 3.22. Let $(\mathcal{A},\{.,\}$.$) be a Poisson algebra generated by two elements x, y$. Again, it follows that $\mathcal{K}=\left(\mathcal{A}, g,\left\{x^{1}, x^{2}\right\}\right)=(\mathcal{A}, g,\{x, y\})$ is a Kähler-Poisson algebra for arbitrary symmetric matrices

$$
g=\left(\begin{array}{ll}
g_{11} & g_{12} \\
g_{12} & g_{22}
\end{array}\right),
$$

with

$$
\eta=\left(\{x, y\}^{2} \operatorname{det}(g)\right)^{-1} .
$$

For $\mathcal{K}^{\prime}=\left(\mathcal{A}, h,\left\{y^{1}, y^{2}\right\}\right)=(\mathcal{A}, h,\{x, x+y\})$, one obtains

$$
\mathcal{P}^{\prime}=\left(\begin{array}{cc}
0 & \{x, y\} \\
-\{x, y\} & 0
\end{array}\right) .
$$

It is easy to check that for the symmetric matrix $h$

$$
h=\left(\begin{array}{ll}
h_{11} & h_{12} \\
h_{12} & h_{22}
\end{array}\right),
$$

one obtains

$$
\mathcal{P}^{\prime} h \mathcal{P}^{\prime} h \mathcal{P}^{\prime}=-\{x, y\}^{2} \operatorname{det}(h) \mathcal{P}^{\prime},
$$

giving $\eta^{\prime}=\left(\{x, y\}^{2} \operatorname{det}(h)\right)^{-1}$. Let us try to find $h$ such that $\mathcal{K} \cong \mathcal{K}^{\prime}$. one easily sees that $\mathfrak{g}=\mathfrak{g}^{\prime}$ as in the previous example. To prove that $\mathcal{K} \cong \mathcal{K}^{\prime}$, we need to define the maps $\phi: \mathcal{A} \rightarrow \mathcal{A}$ and $\psi: \mathfrak{g} \rightarrow \mathfrak{g}$. By choosing $\phi=i d$ and $\psi=i d$ we will show that $(\phi, \psi)$ is an isomorphism of Kähler Poisson algebras for a suitable choice of matrix h. Properties (1),(2),(4) in Definition 3.16 are satisfied. To check property (3), one needs to show that

$$
g(\alpha, \beta)=h(\alpha, \beta) .
$$

Starting from the left hand side we get

$$
\begin{aligned}
\phi(g(\alpha, \beta)) & =\sum_{i, j=1}^{2} \alpha\left(x^{i}\right) g_{i j} \beta\left(x^{j}\right) \\
& =\alpha(x) g_{11} \beta(x)+\alpha(x) g_{12} \beta(y)+\alpha(y) g_{21} \beta(x)+\alpha(y) g_{22} \beta(y) .
\end{aligned}
$$

From the right hand side we get

$$
\begin{aligned}
h(\alpha, \beta) & =\sum_{i, j=1}^{2} \alpha\left(y^{i}\right) h_{i j} \beta\left(y^{j}\right) \\
= & \alpha(x) h_{11} \beta(x)+\alpha(x) h_{12} \beta(x)+\alpha(x) h_{12} \beta(y)+\alpha(x) h_{21} \beta(x)+\alpha(y) h_{21} \beta(x) \\
& +\alpha(y) h_{22} \beta(x)+\alpha(y) h_{22} \beta(x)+\alpha(x) h_{22} \beta(y)+\alpha(y) h_{22} \beta(y) .
\end{aligned}
$$


For these two expressions to coincide, we conclude that the symmetric matrix $h$ becomes

$$
h=\left(\begin{array}{cc}
g_{11}-2 g_{12}+g_{22} & g_{12}-g_{22} \\
g_{21}-g_{22} & g_{22}
\end{array}\right) \text {. }
$$

We check that

$$
\operatorname{det}(h)=\operatorname{det}(g)
$$

and setting $\operatorname{det}(h)$ in equation $(3.9$ one get

$$
\mathcal{P}^{\prime} h \mathcal{P}^{\prime} h \mathcal{P}^{\prime}=-\{x, y\}^{2} \operatorname{det}(g) \mathcal{P}^{\prime}
$$

giving $\eta^{\prime}=\left(\{x, y\}^{2} \operatorname{det}(g)\right)^{-1}=\eta$. We conclude that $g(\alpha, \beta)=h(\alpha, \beta)$, which shows that $\mathcal{K} \cong \mathcal{K}^{\prime}$.

Example 3.23. Let $(\mathcal{A},\{.,\}$.$) be a Poisson algebra generated by two elements$ $x^{1}=x, x^{2}=y$. We know that $\mathcal{K}=(\mathcal{A}, g,\{x, y\})$ is a Kähler-Poisson algebra for arbitrary symmetric matrices

$$
g=\left(\begin{array}{ll}
g_{11} & g_{12} \\
g_{12} & g_{22}
\end{array}\right)
$$

with

$$
\eta=\left(\{x, y\}^{2} \operatorname{det}(g)\right)^{-1}
$$

For $\mathcal{K}^{\prime}=(\mathcal{A}, h,\{x+y, x-y\})$, one obtains

$$
\mathcal{P}^{\prime}=\left(\begin{array}{cc}
0 & -2\{x, y\} \\
2\{x, y\} & 0
\end{array}\right) .
$$

It is easy to check that for the symmetric matrix $h$

$$
h=\left(\begin{array}{ll}
h_{11} & h_{12} \\
h_{12} & h_{22}
\end{array}\right),
$$

one obtains

$$
\mathcal{P}^{\prime} h \mathcal{P}^{\prime} h \mathcal{P}^{\prime}=-4\{x, y\}^{2} \operatorname{det}(h) \mathcal{P}^{\prime},
$$

giving $\eta^{\prime}=\left(4\{x, y\}^{2} \operatorname{det}(h)\right)^{-1}$. Let us try to find $h$ such that $\mathcal{K} \cong \mathcal{K}^{\prime}$. One sees that $\mathfrak{g}=\mathfrak{g}^{\prime}$ as in the previous examples. To prove that $\mathcal{K} \cong \mathcal{K}^{\prime}$, we need to define the maps $\phi: \mathcal{A} \rightarrow \mathcal{A}$ and $\psi: \mathfrak{g} \rightarrow \mathfrak{g}$. By choosing $\phi=i d$ and $\psi=i d$ we will show that $(\phi, \psi)$ is an isomorphism of Kähler Poisson algebras for a suitable choice of matrix h. Properties (1),(2),(4) in Definition 3.16 are satisfied. To check property (3), one needs to show that

$$
g(\alpha, \beta)=h(\alpha, \beta)
$$


Starting from the left hand side we get

$$
\begin{aligned}
g(\alpha, \beta) & =\alpha\left(x^{i}\right) g_{i j} \beta\left(x^{j}\right) \\
& =\alpha(x) g_{11} \beta(x)+\alpha(x) g_{12} \beta(y)+\alpha(y) g_{21} \beta(x)+\alpha(y) g_{22} \beta(y) .
\end{aligned}
$$

From the right hand side we get

$$
\begin{aligned}
& h(\alpha, \beta)=\alpha\left(x^{i}\right) h_{i j} \beta\left(x^{j}\right) \\
& =\alpha(x) h_{11} \beta(x)+\alpha(x) h_{11} \beta(y)+\alpha(y) h_{11} \beta(x)+\alpha(y) h_{11} \beta(y)+2 \alpha(x) h_{12} \beta(x) \\
& \quad-2 \alpha(y) h_{12} \beta(y)+\alpha(x) h_{22} \beta(x)-\alpha(x) h_{22} \beta(y)-\alpha(y) h_{22} \beta(x)+\alpha(y) h_{22} \beta(y) .
\end{aligned}
$$

Therefore, the symmetric matrix $h$ becomes

$$
h=\frac{1}{4}\left(\begin{array}{cc}
g_{11}+2 g_{12}+g_{22} & g_{11}-g_{22} \\
g_{11}-g_{22} & g_{11}-2 g_{12}+g_{22}
\end{array}\right),
$$

giving

$$
\operatorname{det}(h)=\frac{1}{4} \operatorname{det}(g)
$$

Setting $\operatorname{det}(h)$ in equation (3.10) one obtains

$$
\mathcal{P}^{\prime} h \mathcal{P}^{\prime} h \mathcal{P}^{\prime}=-\{x, y\}^{2} \operatorname{det}(g) \mathcal{P}^{\prime}
$$

giving $\eta^{\prime}=\left(\{x, y\}^{2} \operatorname{det}(g)\right)^{-1}=\eta$. We conclude that $g(\alpha, \beta)=h(\alpha, \beta)$, which shows that $\mathcal{K} \cong \mathcal{K}^{\prime}$.

The above examples show explicitly how isomorphic finitely generated KählerPoisson algebras may be presented in many different ways, due to different choices of generators.

\subsection{Subalgebras of Kähler-Poisson algebras}

After having defined Kähler-Poisson algebras it is natural to consider subalgebras of Kähler-Poisson algebras, which are defined below by using the concept of morphism from Definition 3.16. Moreover, we show that the image of a Kähler-Poisson algebra morphism is a Kähler-Poisson algebra.

Definition 3.24. Let $\mathcal{K}=\left(\mathcal{A}, g,\left\{x^{1}, \ldots, x^{m}\right\}\right)$ and $\mathcal{K}^{\prime}=\left(\mathcal{A}^{\prime}, g^{\prime},\left\{y^{1}, \ldots, y^{m^{\prime}}\right\}\right)$ be Kähler-Poisson algebras together with their corresponding modules of derivations $\mathfrak{g}$ and $\mathfrak{g}^{\prime}$, respectively. $\mathcal{K}$ is a Kähler-Poisson subalgebra of $\mathcal{K}^{\prime}$ if:

(A) $\mathcal{A}$ is a Poisson subalgebra of $\mathcal{A}^{\prime}$,

(B) (id $\left.\left.\right|_{\mathcal{A}},\left.\mathrm{id}\right|_{\mathfrak{g}}\right)$ is a homomorphism of Kähler-Poisson algebras, where id $\left.\right|_{\mathcal{A}}$ and id $\left.\right|_{\mathfrak{g}}$ denote the identity maps restricted to $\mathcal{A}$ and $\mathfrak{g}$, respectively. 
Given two Kähler-Poisson algebras $\mathcal{K}$ and $\mathcal{K}^{\prime}$, we illustrate with examples when $\mathcal{K}$ is a Kähler-Poisson subalgebra of $\mathcal{K}^{\prime}$, where $\mathcal{A}$ is a proper Poisson subalgebra of a finitely generated Poisson algebra $\mathcal{A}^{\prime}$. Note that the property (B) in Definition 3.24 determines the metric $g$ on $\mathcal{K}$.

Example 3.25. Let $\mathcal{K}=(\mathcal{A}, g,\{x, y\})$ and $\mathcal{K}^{\prime}=\left(\mathcal{A}^{\prime}, g^{\prime},\{x, y, z\}\right)$ be KählerPoisson algebras, where $\mathcal{A}$ is a subalgebra of $\mathcal{A}^{\prime}$ generated by $\{x, y\}$ and $\mathcal{A}^{\prime}$ generated by $\{x, y, z\}$ such that $\{x, y\}=p(x, y)$ and $\{x, z\}=\{y, z\}=0$. Let us now determine the metric $g$ (in terms of $g^{\prime}$ ) such that $\mathcal{K}$ is a Kähler-Poisson subalgebra of $\mathcal{K}^{\prime}$. Since $\mathcal{A}$ is a Poisson subalgebra of $\mathcal{A}^{\prime}$, to show that $\mathcal{K}$ is a Kähler-Poisson subalgebra of $\mathcal{K}^{\prime}$, we only need to show that $\left(\left.\mathrm{id}\right|_{\mathcal{A}}\right.$, id $\left.\left.\right|_{\mathfrak{g}}\right)$ satisfies the properties in Definition 3.16. This fact determines the metric $g$ on $\mathcal{K}$. The only non-trivial condition is (3). We see that

$$
\phi(g(\alpha, \beta))=g^{\prime}(\psi(\alpha), \psi(\beta))
$$

since

$$
\phi(g(\alpha, \beta))=\alpha(x) g_{11} \beta(x)+\alpha(x) g_{12} \beta(y)+\alpha(y) g_{21} \beta(x)+\alpha(y) g_{22} \beta(y),
$$

and

$$
\begin{aligned}
& g^{\prime}(\psi(\alpha), \psi(\beta))=g^{\prime}(\alpha, \beta)=\sum_{i, j=1}^{3} \alpha\left(x^{i}\right) g_{i j}^{\prime} \beta\left(x^{j}\right) \\
& \quad=\alpha(x) g_{11}^{\prime} \beta(x)+\alpha(x) g_{12}^{\prime} \beta(y)+\alpha(y) g_{21}^{\prime} \beta(x)+\alpha(y) g_{22}^{\prime} \beta(y),
\end{aligned}
$$

since $\alpha=a_{i}\left\{x^{i},.\right\}=a_{1}\{x,\}+.a_{2}\{y,.\} \in \mathfrak{g}$, giving $\alpha(z)=a_{1}\{x, z\}+a_{2}\{y, z\}=0$.

Similarly, $\beta(z)=0$. By comparing both sides $\phi(g(\alpha, \beta))$ and $g^{\prime}(\psi(\alpha), \psi(\beta))$, we conclude that $g_{11}=g_{11}^{\prime}, g_{12}=g_{12}^{\prime}, g_{21}=g_{21}^{\prime}$ and $g_{22}=g_{22}^{\prime}$

$$
g=\left(\begin{array}{ll}
g_{11}^{\prime} & g_{12}^{\prime} \\
g_{21}^{\prime} & g_{22}^{\prime}
\end{array}\right)
$$

Therefore, $\phi(g(\alpha, \beta))=g^{\prime}(\psi(\alpha), \psi(\beta))$. Therefore, $\mathcal{K}$ is a Kähler-Poisson subalgebra of $\mathcal{K}^{\prime}$ if is $g$ obtained from $g^{\prime}$ as above.

Let us take another example with different number of generators.

Example 3.26. Let $\mathcal{K}=(\mathcal{A}, g,\{x, y\})$ and $\mathcal{K}^{\prime}=\left(\mathcal{A}^{\prime}, g^{\prime},\{x, y, z, w\}\right)$ be KählerPoisson algebras, where $\mathcal{A}$ is a subalgebra of $\mathcal{A}^{\prime}$ generated by $\{x, y\}$ and $\mathcal{A}^{\prime}$ generated by $\{x, y, z, w\}$ with $\{x, y\}=p(x, y),\{z, w\}=q(z, w)$ and $\{x, z\}=\{x, w\}=$ $\{y, z\}=\{y, w\}=0$. Let us now determine the metric $g$ (in terms of $g^{\prime}$ ) such that $\mathcal{K}$ is a Kähler-Poisson subalgebra of $\mathcal{K}^{\prime}$. To show that $\mathcal{K}$ is a Kähler-Poisson subalgebra of $\mathcal{K}^{\prime}$ we need to show that $\left(\left.\mathrm{id}\right|_{\mathcal{A}}\right.$, id $\left.\left.\right|_{\mathfrak{g}}\right)$ satisfies the properties in Definition 3.16. The only non-trivial condition is (3). We show that $\phi(g(\alpha, \beta))=$ $g^{\prime}(\psi(\alpha), \psi(\beta))$. The left hand side is

$$
\phi(g(\alpha, \beta))=\alpha(x) g_{11} \beta(x)+\alpha(x) g_{12} \beta(y)+\alpha(y) g_{21} \beta(x)+\alpha(y) g_{22} \beta(y),
$$


and the right hand side is

$$
\begin{aligned}
g^{\prime}(\psi(\alpha), \psi(\beta)) & =g^{\prime}(\alpha, \beta) \\
& =\alpha(x) g_{11}^{\prime} \beta(x)+\alpha(x) g_{12}^{\prime} \beta(y)+\alpha(y) g_{21}^{\prime} \beta(x)+\alpha(y) g_{22}^{\prime} \beta(y),
\end{aligned}
$$

since, $\alpha=a_{i}\left\{x^{i},.\right\}=a_{1}\{x,\}+.a_{2}\{y,.\} \in \mathfrak{g}$, we get $\alpha(z)=a_{1}\{x, z\}+a_{2}\{y, z\}=0$.

Similarly, $\alpha(w)=\beta(z)=\beta(w)=0$. By comparing both sides $\phi(g(\alpha, \beta))$ and $g^{\prime}(\psi(\alpha), \psi(\beta))$, we conclude that $g_{11}=g_{11}^{\prime}, g_{12}=g_{12}^{\prime}, g_{21}=g_{21}^{\prime}$ and $g_{22}=g_{22}^{\prime}$

$$
g=\left(\begin{array}{ll}
g_{11}^{\prime} & g_{12}^{\prime} \\
g_{21}^{\prime} & g_{22}^{\prime}
\end{array}\right)
$$

Therefore, $\phi(g(\alpha, \beta))=g^{\prime}(\psi(\alpha), \psi(\beta))$.

(4) By construction we have that $\phi\left(\mathcal{A}_{\text {fin }}\right) \subseteq \mathcal{A}_{\text {fin }}^{\prime}$.

Therefore, $\mathcal{K}$ is a Kähler-Poisson subalgebra of $\mathcal{K}^{\prime}$ if $g$ is obtained from $g^{\prime}$ as above.

Above we have given examples of when $\mathcal{K}=\left(\mathcal{A}, g,\left\{x^{1}, \ldots, x^{m}\right\}\right)$ is a KählerPoisson subalgebra of $\mathcal{K}^{\prime}=\left(\mathcal{A}^{\prime}, g^{\prime},\left\{y^{1}, \ldots, y^{m \prime}\right\}\right)$, where $\mathcal{A}$ is a Poisson subalgebra of $\mathcal{A}^{\prime}$. The next result shows that one may construct a Kähler-Poisson algebra from the image of a morphism $(\phi, \psi): \mathcal{K} \rightarrow \mathcal{K}^{\prime}$ in the following way.

Proposition 3.27. Let $\mathcal{K}=\left(\mathcal{A}, g,\left\{x^{1}, \ldots, x^{m}\right\}\right)$ and $\mathcal{K}^{\prime}=\left(\mathcal{A}^{\prime}, g^{\prime},\left\{y^{1}, \ldots, y^{m^{\prime}}\right\}\right)$ be Kähler-Poisson algebras, and let $(\phi, \psi): \mathcal{K} \rightarrow \mathcal{K}^{\prime}$ be a homomorphism of KählerPoisson algebras. If $y^{J} \in \phi(\mathcal{A})$ for $J=1, \ldots, m^{\prime}$ then

$$
\operatorname{Im}(\phi, \psi)=\left(\phi(\mathcal{A}), \tilde{g},\left\{\phi\left(x^{1}\right), \phi\left(x^{2}\right), \ldots, \phi\left(x^{m}\right)\right\}\right)
$$

is a Kähler-Poisson subalgebra of $\mathcal{K}^{\prime}$, where

$$
\tilde{g}_{k l}=\phi\left(\eta \mathcal{P}_{k m}\right)\left\{\phi\left(x^{m}\right), y^{J}\right\}^{\prime} g_{J M}^{\prime} \phi\left(\eta \mathcal{P}_{l n}\right)\left\{\phi\left(x^{n}\right), y^{M}\right\}^{\prime} .
$$

\subsection{Direct sums and tensor products of Kähler- Poisson algebras}

Let $\mathcal{K}=\left(\mathcal{A}, g,\left\{x^{1}, \ldots, x^{m}\right\}\right)$ and $\mathcal{K}^{\prime}=\left(\mathcal{A}^{\prime}, g^{\prime},\left\{y^{1}, \ldots, y^{m \prime}\right\}\right)$ be Kähler-Poisson algebras. In this section, we are interested in defining operations of Kähler-Poisson algebras. In particular, we introduce direct sums and tensor products of KählerPoisson algebras and study properties of these operations. We prove that, direct sums and tensor products of two Kähler-Poisson algebras are Kähler-Poisson algebras and it is shown that $\mathcal{K}$ and $\mathcal{K}^{\prime}$ are subalgebras of the direct sum $\mathcal{K} \oplus \mathcal{K}^{\prime}$. We refer to Section 3.1 for the basic notions of direct sums and tensor products of Poisson algebras. In next proposition, the direct sum of two Kähler-Poisson algebras is defined, and we show that it is indeed a Kähler-Poisson algebra.

Proposition 3.28. Let $\mathcal{K}=\left(\mathcal{A}, g,\left\{x^{1}, \ldots, x^{m}\right\}\right)$ and $\mathcal{K}^{\prime}=\left(\mathcal{A}^{\prime}, g^{\prime},\left\{y^{1}, \ldots, y^{m \prime}\right\}\right)$ be Kähler-Poisson algebras, and set $\mathcal{K} \oplus \mathcal{K}^{\prime}=\left(\mathcal{A} \oplus \mathcal{A}^{\prime}, \hat{g},\left\{z^{1}, \ldots, z^{m+m^{\prime}}\right\}\right)$ where

$$
z^{I}= \begin{cases}\left(x^{I}, 0\right) & \text { if } I \in\{1, \ldots, m\} \\ \left(0, y^{I-m}\right) & \text { if } I \in\left\{m+1, \ldots, m+m^{\prime}\right\},\end{cases}
$$


and

$$
\hat{g}_{I J}= \begin{cases}\left(g_{I J}, 0\right) & \text { if } I, J \in\{1, \ldots, m\} \\ \left(0, g_{I-m, J-m}^{\prime}\right) & \text { if } I, J \in\left\{m+1, \ldots, m+m^{\prime}\right\} \\ (0,0) & \text { otherwise. }\end{cases}
$$

Then $\mathcal{K} \oplus \mathcal{K}^{\prime}$ is a Kähler-Poisson algebra.

A natural property of the direct sum of algebras is that each factor is a subalgebra of the direct sum. The next proposition shows that this result is true also for Kähler-Poisson algebras. However, let us first introduce a concrete description of the direct sum of the modules of inner derivations.

Remark 3.29. The module of inner derivations of $\mathcal{A} \oplus \mathcal{A}^{\prime}$ can be written as:

$$
\mathfrak{g} \oplus \mathfrak{g}^{\prime}=\left\{(\alpha, \beta): \alpha \in \mathfrak{g} \text { and } \beta \in \mathfrak{g}^{\prime}\right\}
$$

since given $\tilde{\alpha} \in \mathfrak{g} \oplus \mathfrak{g}^{\prime}$

$$
\begin{aligned}
\tilde{\alpha}(c, d)=\sum_{I=1}^{m+m^{\prime}}\left(a_{I}, b_{I}\right)\left\{z^{I},(c, d)\right\} & =\sum_{I=1}^{m}\left(a_{I}\left\{x^{I}, c\right\}, 0\right)+\sum_{I=m+1}^{m+m^{\prime}}\left(0, b_{I}\left\{y^{I-m}, d\right\}\right) \\
& =(\alpha(c), \beta(d))=(\alpha, \beta)(c, d),
\end{aligned}
$$

with

$$
\alpha=\sum_{I=1}^{m} a_{I}\left\{x^{I}, \cdot\right\} \quad \text { and } \quad \beta=\sum_{I=1}^{m^{\prime}} b_{I+m}\left\{y^{I}, \cdot\right\} .
$$

Proposition 3.30. Let $\mathcal{K}=\left(\mathcal{A}, g,\left\{x^{1}, \ldots, x^{m}\right\}\right)$ and $\mathcal{K}^{\prime}=\left(\mathcal{A}^{\prime}, g^{\prime},\left\{y^{1}, \ldots, y^{m \prime}\right\}\right)$ be Kähler-Poisson algebras, then $\mathcal{K}$ and $\mathcal{K}^{\prime}$ are Kähler-Poisson subalgebras of $\mathcal{K} \oplus \mathcal{K}^{\prime}$.

To define the tensor product of Kähler-Poisson algebras, one has to take a little bit more care. Recall that in the geometric situation of Kähler manifolds, $\eta$ is given as a positive function (in fact, as the square of a real function), implying that $\sqrt{\eta}$ exists. However, in the purely algebraic setting, this is not necessarily true. The next proposition shows that the tensor product of Kähler-Poisson algebras can be defined under the assumption that $\sqrt{\eta}$ exists in the algebra.

Proposition 3.31. Let $\mathcal{K}=\left(\mathcal{A}, g,\left\{x^{1}, \ldots, x^{m}\right\}\right)$ and $\mathcal{K}^{\prime}=\left(\mathcal{A}^{\prime}, g^{\prime},\left\{y^{1}, \ldots, y^{m^{\prime}}\right\}\right)$ with

$$
\sum_{i, j, k, l=1}^{m} \eta\left\{a_{1}, x^{i}\right\} g_{i j}\left\{x^{j}, x^{k}\right\} g_{k l}\left\{x^{l}, b_{1}\right\}=-\left\{a_{1}, b_{1}\right\}
$$

and

$$
\sum_{\alpha, \beta, \gamma, \delta=1}^{m^{\prime}} \eta^{\prime}\left\{a_{2}, y^{\alpha}\right\}^{\prime} g_{\alpha \beta}^{\prime}\left\{y^{\beta}, y^{\gamma}\right\}^{\prime} g_{\gamma \delta}^{\prime}\left\{y^{\delta}, b_{2}\right\}^{\prime}=-\left\{a_{2}, b_{2}\right\}^{\prime}
$$


be Kähler-Poisson algebras, and assume that there exist $\rho \in \mathcal{A}$ and $\rho^{\prime} \in \mathcal{A}^{\prime}$ such that $\rho^{2}=\eta$ and $\rho^{\prime 2}=\eta^{\prime}$. Set $\mathcal{K} \otimes \mathcal{K}^{\prime}=\left(\mathcal{A} \otimes \mathcal{A}^{\prime}, \tilde{g},\left\{z^{1}, \ldots, z^{m+m^{\prime}}\right\}\right)$ where

$$
z^{I}= \begin{cases}x^{I} \otimes 1 & \text { if } I \in\{1, \ldots, m\} \\ 1 \otimes y^{I-m} & \text { if } I \in\left\{m+1, \ldots, m+m^{\prime}\right\},\end{cases}
$$

and

$$
\tilde{g}_{I J}= \begin{cases}\rho g_{I J} \otimes 1 & \text { if } I, J \in\{1, \ldots, m\} \\ 1 \otimes \rho^{\prime} g_{I-m, J-m}^{\prime} & \text { if } I, J \in\left\{m+1, \ldots, m+m^{\prime}\right\} \\ 0 & \text { otherwise. }\end{cases}
$$

Then $\mathcal{K} \otimes \mathcal{K}^{\prime}$ is a Kähler-Poisson algebra.

\subsection{Construction of Kähler-Poisson algebras}

Given a Poisson algebra $\{\mathcal{A},\{.,\}$.$\} one may ask if there exist \left\{x^{1}, \ldots, x^{m}\right\}$ and $\left(g_{i j}\right)$ such that $\left\{\mathcal{A},\left\{x^{1}, \ldots, x^{m}\right\}, g\right\}$ is a Kähler-Poisson algebra? In the previous section we have seen that Poisson algebras generated by two or three variables can always (up to zero-divisors) be localized such that the resulting algebra is a Kähler-Poisson algebra. In this section we will show that a similar result holds true for a large class of Poisson algebras, by outlining a construction procedure which yields a metric inducing a Kähler-Poisson algebra structure for a given Poisson algebra.

Let $\mathcal{A}$ be a finitely generated algebra, and let $\left\{x^{1}, \ldots, x^{m}\right\}$ be an arbitrary set of generators. As usual, denoting by $\mathcal{P}$ the matrix with entries $\left\{x^{i}, x^{j}\right\}$ and by $g$ the symmetric matrix with entries $g_{i j}$, the Kähler-Poisson condition 3.3 is written in matrix notation as

$$
\eta \mathcal{P} g \mathcal{P} g \mathcal{P}=-\mathcal{P}
$$

In the following, we provide a rather general way to associate a localization $\mathcal{A}\left[\lambda^{-1}\right]$ and a metric $g$ to $\mathcal{A}$, such that $\left(\mathcal{A}\left[\lambda^{-1}\right],\left\{x^{1}, \ldots, x^{m}\right\}, g\right)$ is a Kähler-Poisson algebra. To this end, we start by proving a general result for anti-symmetric matrices with entries in a commutative ring.

Thus, let $P$ be an arbitrary antisymmetric matrix with entries in a commutative ring $R$. We will start by writing $P$ as being similar to a block diagonal matrix. This is a well known result in linear algebra, in which case the eigenvalues appear in the diagonal blocks. For an antisymmetric matrix with entries in a commutative ring, a similar result holds.

Proposition 3.32 ([3]). Let $R$ be a commutative ring and let $M_{N}(R)$ denote the set of $N \times N$ matrices with entries in $R$. Moreover, let $P \in M_{N}(R)$ be an antisymmetric matrix, and let $\hat{N}$ denote the integer part of $N / 2$. Then there exists $V \in M_{N}(R)$ and $\lambda_{1}, \ldots, \lambda_{\hat{N}} \in R$ such that

$$
\begin{aligned}
& V^{T} P V=\operatorname{diag}\left(\Lambda_{1}, \ldots, \Lambda_{\hat{N}}\right) \quad \text { if } N \text { is even } \\
& V^{T} P V=\operatorname{diag}\left(\Lambda_{1}, \ldots, \Lambda_{\hat{N}}, 0\right) \quad \text { if } N \text { is odd, }
\end{aligned}
$$


where

$$
\Lambda_{k}=\left(\begin{array}{cc}
0 & \lambda_{k} \\
-\lambda_{k} & 0
\end{array}\right)
$$

Returning to the case of a Poisson algebra generated by $x^{1}, \ldots, x^{m}$, assume for the ease of presentation that $m=2 N$ for a positive integer $N$. By Proposition 3.32 , there exists a matrix $V$ such that

$$
V^{T} \mathcal{P} V=\mathcal{P}_{0}
$$

where $\mathcal{P}_{0}$ is a block diagonal matrix of the form

$$
\mathcal{P}_{0}=\operatorname{diag}\left(\Lambda_{1}, \ldots, \Lambda_{N}\right)
$$

with

$$
\Lambda_{k}=\left(\begin{array}{cc}
0 & \lambda_{k} \\
-\lambda_{k} & 0
\end{array}\right)
$$

Similarly, define $\lambda=\lambda_{1} \cdots \lambda_{N}$ and $g_{0}=\operatorname{diag}\left(g_{1}, \ldots, g_{N}\right)$ with

$$
g_{k}=\frac{\lambda}{\lambda_{k}}\left(\begin{array}{ll}
1 & 0 \\
0 & 1
\end{array}\right)
$$

for $k=1, \ldots, N$ and set $g=V g_{0} V^{T}$. Noticing that

$$
\mathcal{P}_{0} g_{0} \mathcal{P}_{0} g_{0} \mathcal{P}_{0}=-\lambda^{2} \mathcal{P}_{0}
$$

one finds

$$
0=\mathcal{P}_{0} g_{0} \mathcal{P}_{0} g_{0} \mathcal{P}_{0}+\lambda^{2} \mathcal{P}_{0}=V^{T}\left(\mathcal{P} g \mathcal{P} g \mathcal{P}+\lambda^{2} \mathcal{P}\right) V
$$

It is a general fact that for an arbitrary matrix $V$ there exists a matrix $\tilde{V}$ such that $\tilde{V} V=V \tilde{V}=(\operatorname{det} V) \mathbb{1}$. Multiplying the above equation from the left by $\tilde{V}^{T}$ and from the right by $\tilde{V}$ yields

$$
\operatorname{det}(V)^{2}\left(\mathcal{P} g \mathcal{P} g \mathcal{P}+\lambda^{2} \mathcal{P}\right)=0
$$

As long as $\operatorname{det}(V)$ is not a zero divisor, this implies that

$$
\mathcal{P} g \mathcal{P} g \mathcal{P}=-\lambda^{2} \mathcal{P}
$$

Now, if $\lambda^{2}$ is invertible, then the above procedure yields a Kähler-Poisson algebra $\left(\mathcal{A}, g,\left\{x^{1}, \ldots, x^{m}\right\}\right.$ with $\eta=\lambda^{-2}$. Otherwise, one may localize $\mathcal{A}$ such that $\left(\mathcal{A}\left[\lambda^{-1}\right], g,\left\{x^{1}, \ldots, x^{m}\right\}\right)$ is a Kähler Poisson algebra. 


\section{4 - Curvature and isomorphism classes}

\subsection{Levi-Civita connections and curvature}

In Riemannian geometry, the Levi-Civita connection is a specific connection on the tangent bundle of a manifold. More precisely, it is the torsion-free metric connection, i.e., the torsion-free connection on the tangent bundle preserving a given (pseudo-)Riemannian metric. The fundamental theorem of Riemannian geometry states that there is a unique connection which satisfies these properties above (see e.g. [10]).

In the theory of Riemannian and pseudo-Riemannian manifolds the term covariant derivative is often used for the Levi-Civita connection. The components of this connection with respect to a system of local coordinates are called Christoffel symbols. In Chapter 2 we showed that one can generalize the Levi-Civita connection to metric Lie-Rinehart algebras and so also for Kähler-Poisson algebras. In this section, we shall derive an explicit expression for the Levi-Civita connection of an arbitrary Kähler-Poisson algebra $\mathcal{K}=\left(\mathcal{A}, g,\left\{x^{1}, \cdots, x^{m}\right\}\right)$. Recall that for a Kähler-Poisson algebra, the Levi-Civita connection is the unique torsion-free and metric connection on the module $\mathfrak{g}$.

In the following, it turns out to be convenient to reformulate the results in terms of the generators $\left\{\mathcal{D}^{1}, \cdots, \mathcal{D}^{m}\right\}$ of $\mathfrak{g}$ (cf. Proposition 3.13). Applying Koszul's formula in Proposition 2.11 gives the connection as

$$
\begin{array}{r}
2 g\left(\nabla_{\mathcal{D}^{i}} \mathcal{D}^{j}, \mathcal{D}^{k}\right)=\mathcal{D}^{i}\left(g\left(\mathcal{D}^{j}, \mathcal{D}^{k}\right)\right)+\mathcal{D}^{j}\left(g\left(\mathcal{D}^{k}, \mathcal{D}^{i}\right)\right)-\mathcal{D}^{k}\left(g\left(\mathcal{D}^{i}, \mathcal{D}^{j}\right)\right) \\
-g\left(\left[\mathcal{D}^{j}, \mathcal{D}^{k}\right], \mathcal{D}^{i}\right)+g\left(\left[\mathcal{D}^{k}, \mathcal{D}^{i}\right], \mathcal{D}^{j}\right)+g\left(\left[\mathcal{D}^{i}, \mathcal{D}^{j}\right], \mathcal{D}^{k}\right)
\end{array}
$$

and one notes that an element $\alpha=a\{b,$.$\} may be recovered from g\left(\alpha, \mathcal{D}^{i}\right)$ as

$$
g\left(\alpha, \mathcal{D}^{i}\right) \mathcal{D}_{i}(f)=a\left\{b, x^{k}\right\} \mathcal{D}_{k}^{i} \mathcal{D}_{i}(f)=a\left\{b, x^{k}\right\} \mathcal{D}_{k}(f)=a\{b, f\}=\alpha(f) .
$$

Thus, one immediately obtains $\nabla_{\mathcal{D}^{i}} \mathcal{D}^{j}=g\left(\nabla_{\mathcal{D}^{i}} \mathcal{D}^{j}, \mathcal{D}^{k}\right) \mathcal{D}_{k}$. However, it turns out that one can obtain a more convenient expression for the connection. Let us start with the following result.

Lemma 4.1 $([\underline{3}]) \cdot g\left(\left[\mathcal{D}^{i}, \mathcal{D}^{j}\right], \mathcal{D}^{k}\right)=\mathcal{D}^{i}\left(\mathcal{D}^{j k}\right)-\mathcal{D}^{j}\left(\mathcal{D}^{i k}\right)$.

The above result allows for a rather compact formula of the Levi-Civita connection for a Kähler Poisson-algebra. Namely, using Koszul's formula and Lemma 4.1 we obtain the following result.

Proposition $4.2([3])$. If $\nabla$ denotes the Levi-Civita connection of a KählerPoisson algebra $\mathcal{K}$ then

$$
\nabla_{\mathcal{D}^{i}} \mathcal{D}^{j}=\frac{1}{2} \mathcal{D}^{i}\left(\mathcal{D}^{j k}\right) \mathcal{D}_{k}-\frac{1}{2} \mathcal{D}^{j}\left(\mathcal{D}^{i k}\right) \mathcal{D}_{k}+\frac{1}{2} \mathcal{D}^{k}\left(\mathcal{D}^{i j}\right) \mathcal{D}_{k}
$$


or, equivalently, $\nabla_{\mathcal{D}^{i}} \mathcal{D}^{j}=\Gamma^{i j}{ }_{k} \mathcal{D}^{k}$ where

$$
\Gamma_{k}^{i j}=\frac{1}{2} \mathcal{D}^{i}\left(\mathcal{D}^{j l}\right) \mathcal{D}_{l k}-\frac{1}{2} \mathcal{D}^{j}\left(\mathcal{D}^{i l}\right) \mathcal{D}_{l k}+\frac{1}{2} \mathcal{D}_{k}\left(\mathcal{D}^{i j}\right) .
$$

For $\alpha=\alpha_{i} \mathcal{D}^{i}$ and $\beta=\beta_{i} \mathcal{D}^{i}$ arbitrary elements of $\mathfrak{g}$, one obtains

$$
\nabla_{\alpha} \beta=\alpha\left(\beta_{i}\right) \mathcal{D}^{i}+\Gamma_{k}^{i j} \alpha_{i} \beta_{j} \mathcal{D}^{k} .
$$

From Section 2 we recall the curvature

$$
R(\alpha, \beta) \gamma=\nabla_{\alpha} \nabla_{\beta} \gamma-\nabla_{\beta} \nabla_{\alpha} \gamma-\nabla_{[\alpha, \beta]} \gamma,
$$

as well as

$$
\begin{aligned}
R(\alpha, \beta, \gamma) & =R(\alpha, \beta) \gamma \\
R(\alpha, \beta, \gamma, \delta) & =g(\alpha, R(\gamma, \delta) \beta) .
\end{aligned}
$$

For the sake of illustration, let us consider an example where we compute the curvature in detail.

Example 4.3. Let $\mathcal{K}$ be a Kähler-Poisson algebra generated by two elements

$$
\mathcal{K}=(\mathcal{A}, g,\{x, y\})
$$

with

$$
g=\frac{1}{\lambda}\left(\begin{array}{ll}
1 & 0 \\
0 & 1
\end{array}\right)
$$

where $\lambda$ is assumed to be an invertible element of $\mathcal{A}$. From previous considerations, we know that $(\mathcal{A}, g,\{x, y\})$ is a Kähler-Poisson algebra with $\eta=\frac{\lambda^{2}}{p^{2}}$ and $p=\{x, y\}$. We introduce $\gamma=\frac{p}{\lambda}$ such that $\eta=\frac{1}{\gamma^{2}}$. Let us start by computing the derivations $\mathcal{D}^{x}=\mathcal{D}^{1}$ and $\mathcal{D}^{y}=\mathcal{D}^{2}$, which generate the module $\mathfrak{g}$ :

$$
\begin{aligned}
& \mathcal{D}^{x}=\eta\left\{x, x^{i}\right\} g_{i j}\left\{\cdot, y^{i}\right\}=\frac{\lambda}{p}\{\cdot, y\}=\frac{1}{\gamma}\{\cdot, y\} \\
& \mathcal{D}^{y}=\eta\left\{y, x^{i}\right\} g_{i j}\left\{\cdot, x^{i}\right\}=\frac{\lambda}{p}\{\cdot, x\}=-\frac{1}{\gamma}\{\cdot, x\}
\end{aligned}
$$

as well as

$$
\mathcal{D}_{x}=g_{1 k} \mathcal{D}^{k}=\frac{1}{\lambda} \mathcal{D}^{x} \quad \text { and } \quad \mathcal{D}_{y}=g_{2 k} \mathcal{D}^{k}=\frac{1}{\lambda} \mathcal{D}^{y}
$$

Moreover, they provide an orthogonal set of generators since

$$
g\left(\mathcal{D}^{x}, \mathcal{D}^{x}\right)=g\left(\mathcal{D}^{y}, \mathcal{D}^{y}\right)=\lambda
$$

and $g\left(\mathcal{D}^{x}, \mathcal{D}^{y}\right)=0$. Let us define the derivation $\mathcal{D}^{\lambda}=\gamma^{-1}\{\lambda, \cdot\}$ and note that

$$
\mathcal{D}^{\lambda}=\left[\mathcal{D}^{x}, \mathcal{D}^{y}\right]=\frac{1}{\lambda} \mathcal{D}^{x}(\lambda) \mathcal{D}^{y}-\frac{1}{\lambda} \mathcal{D}^{y}(\lambda) \mathcal{D}^{x} .
$$


One can compute

$$
g\left(\nabla_{\mathcal{D}^{x}} \mathcal{D}^{y}, \mathcal{D}^{x}\right)=\frac{1}{2 \gamma}\{\lambda, x\} \quad \text { and } \quad g\left(\nabla_{\mathcal{D}^{x}} \mathcal{D}^{y}, \mathcal{D}^{y}\right)=\frac{1}{2 \gamma}\{\lambda, y\} .
$$

Therefore,

$$
\left(\nabla_{\mathcal{D}^{x}} \mathcal{D}^{y}\right)(f)=\frac{1}{2} \mathcal{D}^{x}(\lambda) \mathcal{D}_{y}-\frac{1}{2} \mathcal{D}^{y}(\lambda) \mathcal{D}_{x}
$$

since $\mathcal{D}^{x}(\lambda)=\frac{1}{\gamma}\{\lambda, y\}, \mathcal{D}^{y}(\lambda)=-\frac{1}{\gamma}\{\lambda, x\}$ and $\mathcal{D}_{y}=\frac{1}{\lambda} \mathcal{D}^{y}(\lambda)$. Similarly,

$$
\begin{aligned}
\nabla_{\mathcal{D}^{y}} \mathcal{D}^{y} & =\frac{1}{2} \mathcal{D}^{x}(\lambda) \mathcal{D}_{x}+\frac{1}{2} \mathcal{D}^{y}(\lambda) \mathcal{D}_{y} \\
\nabla_{\mathcal{D}^{x}} \mathcal{D}^{x} & =\frac{1}{2} \mathcal{D}^{x}(\lambda) \mathcal{D}_{x}+\frac{1}{2} \mathcal{D}^{y}(\lambda) \mathcal{D}_{y} \\
\nabla_{\mathcal{D}^{y}} \mathcal{D}^{x} & =\frac{1}{2} \mathcal{D}^{y}(\lambda) \mathcal{D}_{x}-\frac{1}{2} \mathcal{D}^{x}(\lambda) \mathcal{D}_{y}
\end{aligned}
$$

Moreover, the curvature can be computed

$$
R\left(\mathcal{D}^{x}, \mathcal{D}^{y}\right) \mathcal{D}^{x}=\left[\mathcal{D}_{x}(\lambda)^{2}+\mathcal{D}_{y}(\lambda)^{2}-\frac{1}{2} \mathcal{D}_{x}\left(\mathcal{D}^{x}(\lambda)\right)-\frac{1}{2} \mathcal{D}_{y}\left(\mathcal{D}^{y}(\lambda)\right)\right] \mathcal{D}^{y}
$$

and

$$
R\left(\mathcal{D}^{x}, \mathcal{D}^{y}\right) \mathcal{D}^{y}=-\left[\mathcal{D}_{x}(\lambda)^{2}+\mathcal{D}_{y}(\lambda)^{2}-\frac{1}{2} \mathcal{D}_{x}\left(\mathcal{D}^{x}(\lambda)\right)-\frac{1}{2} \mathcal{D}_{y}\left(\mathcal{D}^{y}(\lambda)\right)\right] \mathcal{D}^{x} .
$$

\subsection{Properties of isomorphisms}

We are interested in studying properties of isomorphisms for Kähler-Poisson algebras, which we will use in the next section to study moduli spaces. In particular, we ask when two Kähler-Poisson algebras are isomorphic, and what properties they share. Let $\mathcal{K}=\left(\mathcal{A}, g,\left\{x^{1}, \ldots, x^{m}\right\}\right)$ and $\mathcal{K}^{\prime}=\left(\mathcal{A}^{\prime}, g^{\prime},\left\{y^{1}, \ldots, y^{m^{\prime}}\right\}\right)$ be Kähler-Poisson algebras and assume that there exists a Poisson algebra isomorphism $\phi: \mathcal{A} \rightarrow \mathcal{A}^{\prime}$. When does there exist a map $\psi: \mathfrak{g} \rightarrow \mathfrak{g}^{\prime}$ such that $(\phi, \psi)$ is an isomorphism of Kähler-Poisson algebras? The following result provides an answer to this question.

Proposition 4.4. Let $\mathcal{K}=\left(\mathcal{A}, g,\left\{x^{1}, \ldots, x^{m}\right\}\right)$ and $\mathcal{K}^{\prime}=\left(\mathcal{A}^{\prime}, g^{\prime},\left\{y^{1}, \ldots, y^{m^{\prime}}\right\}\right)$ be Kähler-Poisson algebras. $\mathcal{K}$ and $\mathcal{K}^{\prime}$ are isomorphic if and only if there exists a Poisson algebra isomorphism $\phi: \mathcal{A} \rightarrow \mathcal{A}^{\prime}$ such that $\phi\left(\mathcal{A}_{\text {fin }}\right)=\mathcal{A}_{\text {fin }}^{\prime}$, and

$$
\mathcal{P}^{\prime} g^{\prime} \mathcal{P}^{\prime}=\mathcal{P}^{\prime} A^{T} \phi(g) A \mathcal{P}^{\prime}
$$

where $A^{i}{ }_{\alpha}=\frac{\partial \phi\left(x^{i}\right)}{\partial y^{\alpha}}$ and $\left(\mathcal{P}^{\prime}\right)^{\alpha \beta}=\left\{y^{\alpha}, y^{\beta}\right\}$.

As an illustration, let us apply this result to Example 3.23 to again find the metric $h$ such that $\mathcal{K} \cong \mathcal{K}^{\prime}$. 
Example 4.5. From Proposition 4.4 it follows that if we set $h=A^{T} \phi(g) A$, then $\mathcal{P}^{\prime} h \mathcal{P}^{\prime}=\mathcal{P}^{\prime} A^{T} \phi(g) A \mathcal{P}^{\prime}$ implying that $\mathcal{K} \cong \mathcal{K}^{\prime}$.

Let $y^{1}=x+y$ and $y^{2}=x-y$. Hence $x=\frac{1}{2}\left(y^{1}+y^{2}\right)$ and $y=\frac{1}{2}\left(y^{1}-y^{2}\right)$. We compute the matrix A from

$$
A_{\alpha}^{i}=\frac{\partial \phi\left(x^{i}\right)}{\partial y^{\alpha}}=\frac{\partial x^{i}}{\partial y^{\alpha}}
$$

recalling that $\phi=i d$. Therefore, the matrix $A$ becomes

$$
A=\left(\begin{array}{cc}
\frac{1}{2} & \frac{1}{2} \\
\frac{1}{2} & -\frac{1}{2}
\end{array}\right)
$$

and

$$
h=A^{T} \phi(g) A=\left(\begin{array}{cc}
\frac{1}{4}\left(g_{11}+2 g_{12}+g_{22}\right) & \frac{1}{4}\left(g_{11}-g_{22}\right) \\
\frac{1}{4}\left(g_{11}-g_{22}\right) & \frac{1}{4}\left(g_{11}-2 g_{12}+g_{22}\right)
\end{array}\right),
$$

which agrees with the result in Example 3.23. Also $\phi\left(\mathcal{A}_{\text {fin }}\right)=\phi\left(\mathcal{A}_{\text {fin }}^{\prime}\right)$, since $\phi=i d$. Therefore one can now use Proposition 4.4 to conclude that $\mathcal{K} \cong \mathcal{K}^{\prime}$.

In all the examples so far, we have seen that $\eta=\eta^{\prime}$ if $\mathcal{K} \cong \mathcal{K}^{\prime}$. This is a more general fact, which is stated in the following result.

Proposition 4.6. Let $\mathcal{K}=\left(\mathcal{A}, g,\left\{x^{1}, \ldots, x^{m}\right\}\right)$ and $\mathcal{K}^{\prime}=\left(\mathcal{A}^{\prime}, g^{\prime},\left\{y^{1}, \ldots, y^{m^{\prime}}\right\}\right)$ be Kähler-Poisson algebras, and let $(\phi, \psi): \mathcal{K} \rightarrow \mathcal{K}^{\prime}$ be an isomorphism of KählerPoisson algebras. If

$$
\eta \mathcal{P} g \mathcal{P} g \mathcal{P}=-\mathcal{P} \quad \text { and } \quad \eta^{\prime} \mathcal{P}^{\prime} g^{\prime} \mathcal{P}^{\prime} g^{\prime} \mathcal{P}^{\prime}=-\mathcal{P}^{\prime}
$$

then $\left(\phi(\eta)-\eta^{\prime}\right) \mathcal{P}^{\prime}=0$.

Note that if at least one of $\left\{y^{\alpha}, y^{\beta}\right\}$ is not a zero divisor, then Proposition 4.6 implies that $\phi(\eta)=\eta^{\prime}$.

\subsection{Moduli spaces for a class of Kähler-Poisson algebras}

In Section 4.2, some properties of isomorphisms of Kähler-Poisson algebras were presented. Now, we ask the following question: how many different (non isomorphic) choices of a metric structure are there for a fixed Poisson algebra $\mathcal{A}$ ? This is the main theme of Paper III [6]. In general, this is a hard problem and therefore we start from the polynomial algebra $\mathbb{C}[x, y]$ and study the simple case of a Poisson algebra obtained from rational functions in two variables. In principle, we would like to study automorphisms of $\mathbb{C}[x, y]$ but, as shown before, the construction of a Kähler-Poisson algebra over $\mathbb{C}[x, y]$ will in most cases involve a localization of the algebra; therefore, we start by considering rational functions. To this end, we 
let $\mathcal{A}(x, y)=\mathbb{C}(x, y)$ be the rational functions in two variables $x$ and $y$ with Poisson structure given by $\{x, y\}=x$. Although this is a particular choice, one can show that every (non-zero) linear Poisson structure is isomorphic to the one given above [6]. In the following, given the Poisson algebra $\mathcal{A}(x, y)$ we study the possible Kähler-Poisson algebra structures arising from $\mathcal{A}(x, y)$, that is, finding $g$ such that $(\mathcal{A}(x, y), g,\{x, y\})$ is a Kähler-Poisson algebra. We shall repeatedly make use of Proposition 4.4 and Proposition 4.6 to constrain the possible automorphisms of $\mathbb{C}[x, y]$, when studying the isomorphism problem.

Indeed, it is easy to check that for an arbitrary symmetric matrix $g$ with $\operatorname{det}(g) \neq 0$ one obtains

$$
\eta \mathcal{P} g \mathcal{P} g \mathcal{P}=-\{x, y\}^{2} \operatorname{det}(g) \mathcal{P}=-x^{2} \operatorname{det}(g) \mathcal{P}
$$

giving $\eta=\left(x^{2} \operatorname{det}(g)\right)^{-1}$ implying that $(\mathcal{A}(x, y), g,\{x, y\})$ is a Kähler-Poisson algebra. Hence, every non-degenerate $(2 \times 2)$ matrix $g$, with entries in $\mathcal{A}(x, y)$, gives rise to a Kähler-Poisson algebra over $\mathcal{A}(x, y)$. Let us recall that all automorphisms of $\mathbb{C}[x, y]$ (see [15, 21]) are given by compositions of

$$
\phi(x)=\alpha_{1} x+\beta_{1} y+\gamma_{1} \quad \text { and } \quad \phi(y)=\alpha_{2} x+\beta_{2} y+\gamma_{2}
$$

for $\alpha_{1}, \beta_{1}, \gamma_{1}, \alpha_{2}, \beta_{2}, \gamma_{2} \in \mathbb{C}$, with $\alpha_{1} \beta_{2} \neq \alpha_{2} \beta_{1}$ and

$$
\phi(x)=x \quad \text { and } \quad \phi(y)=y+p(x)
$$

for all $p(x) \in \mathbb{C}[x]$. In order to use these to construct Kähler-Poisson algebra morphisms, we need to check which ones that are Poisson algebra morphisms.

Lemma $4.7([6])$. Let $\mathcal{A}(x, y)=\mathbb{C}(x, y)$ be the rational functions in $x, y$ with a Poisson structure given by $\{x, y\}=x$. Then:

(A) $\phi(x)=\alpha_{1} x+\beta_{1} y+\gamma_{1}$ and $\phi(y)=\alpha_{2} x+\beta_{2} y+\gamma_{2}$, for $\alpha_{1}, \beta_{1}, \gamma_{1}, \alpha_{2}, \beta_{2}, \gamma_{2} \in \mathbb{C}$ with $\alpha_{1} \beta_{2} \neq \alpha_{2} \beta_{1}$, is a Poisson algebra automorphism of $\mathcal{A}(x, y)$ if $\beta_{1}=\gamma_{1}=$ 0 and $\beta_{2}=1$, giving $\phi(x)=\alpha_{1} x$ and $\phi(y)=\alpha_{2} x+y+\gamma_{2}$.

(B) $\phi(x)=\alpha x$ and $\phi(y)=y+p(x)$ is a Poisson algebra automorphism for all $p(x) \in \mathbb{C}[x]$ and $\alpha \in \mathbb{C} \backslash\{0\}$.

Note that compositions of Poisson algebra automorphisms in Lemma 4.7 may be written in a simple form.

Proposition $4.8([6])$. Let $\phi=\phi_{1} \circ \phi_{2} \circ \ldots \circ \phi_{n}$ be an arbitrary composition of automorphisms, where each $\phi_{k}$ can be written as: either $\phi(x)=\alpha_{1} x, \phi(y)=$ $\alpha_{2} x+y+\gamma_{2}$ or $\phi(x)=\alpha x, \phi(y)=y+p(x)$. Then there exists $\alpha \in \mathbb{C}$ and $p(x) \in \mathbb{C}[x]$ such that $\phi(x)=\alpha x$ and $\phi(y)=y+p(x)$.

In the next Proposition we explicitly describe all Poisson algebra automorphisms when $\phi(x) \in \mathbb{C}(x)$, where $\mathbb{C}(x)$ denotes the rational functions in $x$. This result will be used in the proofs Proposition 4.10 and Proposition 4.13 to constrain the possible forms of an automorphism. 
Proposition 4.9 (6]). Let $\phi: \mathcal{A}(x, y) \rightarrow \mathcal{A}(x, y)$ be a Poisson algebra automorphism such that $\phi(x) \in \mathbb{C}(x)$. Then there exist $\alpha, \beta, \gamma, \delta \in \mathbb{C}$ and $r(x) \in \mathbb{C}(x)$ such that $\alpha \delta-\beta \gamma \neq 0$ and

$$
\begin{aligned}
& \phi(x)=\frac{\alpha x+\beta}{\gamma x+\delta} \\
& \phi(y)=\frac{(\alpha x+\beta)(\gamma x+\delta) y}{(\alpha \delta-\beta \gamma) x}+r(x)
\end{aligned}
$$

By using the above results, together with Proposition 4.4 and Proposition 4.6, we will now investigate isomorphism classes of metrics for Kähler-Poisson algebras over $\mathcal{A}(x, y)$. The simplest case is when the metrics are constant; i.e: if we take

$$
g=\left(\begin{array}{ll}
a & b \\
b & c
\end{array}\right) \quad \text { and } \quad \tilde{g}=\left(\begin{array}{cc}
\tilde{a} & \tilde{b} \\
\tilde{b} & \tilde{c}
\end{array}\right)
$$

where $a, b, c, \tilde{a}, \tilde{b}, \tilde{c} \in \mathbb{C}$.

Proposition 4.10. Let $\mathcal{K}=(\mathcal{A}(x, y), g,\{x, y\})$ and $\tilde{\mathcal{K}}=(\mathcal{A}(x, y), \tilde{g},\{x, y\})$ be Kähler-Poisson algebras, with

$$
g=\left(\begin{array}{ll}
a & b \\
b & c
\end{array}\right) \quad \text { and } \quad \tilde{g}=\left(\begin{array}{ll}
\tilde{a} & \tilde{b} \\
\tilde{b} & \tilde{c}
\end{array}\right)
$$

where $a, b, c, \tilde{a}, \tilde{b}, \tilde{c} \in \mathbb{C}$ such that $\operatorname{det}(g) \neq 0$ and $\operatorname{det}(\tilde{g}) \neq 0$. Then $\mathcal{K} \cong \tilde{\mathcal{K}}$ if and only if $c=\tilde{c}$.

Let us now say a few words about the proof of Proposition 4.10 . First, we assume that $c=\tilde{c}$. Using an automorphism of the form $\phi(x)=\alpha x$ and $\phi(y)=y+p(x)$ one can apply Proposition 4.4 to explicitly show that there exists $\alpha \in \mathbb{C}$ and $p(x) \in \mathbb{C}[x]$ such that $\phi$ gives and isomorphism between $\mathcal{K}$ and $\tilde{\mathcal{K}}$. For the other direction, we assume that $\mathcal{K} \cong \tilde{\mathcal{K}}$. Then

$$
\eta=\{x, y\}^{2} \operatorname{det}(g)=x^{2} \operatorname{det}(g) \text { and } \tilde{\eta}=\{x, y\}^{2} \operatorname{det}(\tilde{g})=x^{2} \operatorname{det}(\tilde{g})
$$

with $\operatorname{det}(g), \operatorname{det}(\tilde{g}) \in \mathbb{C}$. By using Proposition 4.6. which implies that $\phi(\eta)=\tilde{\eta}$, one obtains

$$
\phi\left(x^{2}\right) \operatorname{det}(g)=x^{2} \operatorname{det}(\tilde{g}) \Rightarrow \frac{\phi\left(x^{2}\right)}{x^{2}}=\frac{\operatorname{det} \tilde{g}}{\operatorname{det}(g)} \in \mathbb{C}
$$

where $\operatorname{det}(g), \operatorname{det}(\tilde{g}) \in \mathbb{C}$, implying that $\phi(x)=\alpha x$ for some $\alpha \in \mathbb{C}$. Applying Proposition 4.9 to such an isomorphism gives $\phi(y)=y+r(x)$. For such an automorphism, Proposition 4.4 via $\tilde{g}=A^{T} \phi(g) A$ immediately gives $c=\tilde{c}$. We remark that a similar line of argument is also the basis for the proof of Proposition 4.13.

In the next result, we study the case when the metric only depends on $x$, and find sufficient conditions for the Kähler-Poisson algebras to be isomorphic. 
Proposition 4.11. Let $\mathcal{K}=(\mathcal{A}(x, y), g,\{x, y\})$ and $\tilde{\mathcal{K}}=(\mathcal{A}(x, y), \tilde{g},\{x, y\})$ be Kähler-Poisson algebras, with

$$
g=\left(\begin{array}{ll}
a(x) & b(x) \\
b(x) & c(x)
\end{array}\right) \quad \text { and } \quad \tilde{g}=\left(\begin{array}{ll}
\tilde{a}(x) & \tilde{b}(x) \\
\tilde{b}(x) & \tilde{c}(x)
\end{array}\right)
$$

where $a(x), b(x), c(x), \tilde{a}(x), \tilde{b}(x), \tilde{c}(x) \in \mathbb{C}[x]$ such that $\operatorname{det}(g) \neq 0$ and $\operatorname{det}(\tilde{g}) \neq 0$. If $\tilde{c}(x) \neq 0$ and there exists $\alpha \in \mathbb{C}$ such that:

1. $\left(\tilde{a}(x)-\alpha^{2} a(\alpha x)\right) c(\alpha x)=\tilde{b}(x)^{2}-\alpha^{2} b(\alpha x)^{2}$

2. $\frac{\tilde{b}(x)-\alpha b(\alpha x)}{c(\alpha x)} \in \mathbb{C}[x]$

3. $\tilde{c}(x)=c(\alpha x)$

then $\mathcal{K} \cong \tilde{\mathcal{K}}$. If $\tilde{c}(x)=c(x)=0$ and there exists $\alpha \in \mathbb{C}$ such that:

(a) $\tilde{b}(x)=\alpha b(\alpha x)$

(b) $\frac{\tilde{a}(x)-\alpha^{2} a(\alpha x)}{2 \alpha b(\alpha x)} \in \mathbb{C}[x]$

then $\mathcal{K} \cong \tilde{\mathcal{K}}$.

Let us use the above result to give a simple example, and construct two seemingly different metrics that give rise to isomorphic Kähler-Poisson algebras.

Example 4.12. Let $\mathcal{K}=(\mathcal{A}(x, y), g,\{x, y\})$ and $\tilde{\mathcal{K}}=(\mathcal{A}(x, y), \tilde{g},\{x, y\})$ be KählerPoisson algebras, with

$$
g=\left(\begin{array}{cc}
a(x) & 0 \\
0 & c(x)
\end{array}\right) \quad \text { and } \quad \tilde{g}=\left(\begin{array}{cc}
a(x)+q(x)^{2} c(x) & q(x) c(x) \\
q(x) c(x) & c(x)
\end{array}\right)
$$

for $a(x), c(x), q(x) \in \mathbb{C}[x]$. We will use Proposition 4.11 to show that $\mathcal{K} \cong \tilde{\mathcal{K}}$. Let us check conditions (1)-(3) with $\alpha=1$.

1.

$$
\begin{aligned}
(\tilde{a}(x)-a(x)) c(x) & =q(x)^{2} c(x)^{2} \\
\tilde{a}(x)-a(x) & =q(x)^{2} c(x) \\
\tilde{a}(x) & =a(x)+q(x)^{2} c(x)
\end{aligned}
$$

2. If $\tilde{b}(x)=q(x) c(x)$ then $\frac{\tilde{b}(x)}{c(x)} \in \mathbb{C}[x]$ for any $q(x) \in \mathbb{C}[x]$.

3. $\tilde{c}(x)=c(x)$.

Therefore, we obtain $\mathcal{K} \cong \tilde{\mathcal{K}}$. For instance, with $a(x)=x, c(x)=x^{2}$ and $q(x)=x^{3}$ one concludes that

$$
g=\left(\begin{array}{cc}
x & 0 \\
0 & x^{2}
\end{array}\right) \text { and }\left(\begin{array}{cc}
x+x^{8} & x^{5} \\
x^{5} & x^{2}
\end{array}\right)
$$

define isomorphic Kähler-Poisson algebras. 
The following result shows that, in the more restricted situation where the metrics are assumed to be diagonal, one can describe all isomorphism classes.

Proposition 4.13. Let $\mathcal{K}=(\mathcal{A}(x, y), g,\{x, y\})$ and $\tilde{\mathcal{K}}=(\mathcal{A}(x, y), \tilde{g},\{x, y\})$ be Kähler-Poisson algebras, with

$$
g=\left(\begin{array}{cc}
a(x) & 0 \\
0 & c(x)
\end{array}\right) \quad \text { and } \quad \tilde{g}=\left(\begin{array}{cc}
\tilde{a}(x) & 0 \\
0 & \tilde{c}(x)
\end{array}\right)
$$

where $a(x), c(x), \tilde{a}(x), \tilde{c}(x) \in \mathbb{C}[x]$ such that $\operatorname{det}(g) \neq 0$ and $\operatorname{det}(\tilde{g}) \neq 0$. Then $\mathcal{K} \cong \tilde{\mathcal{K}}$ if and only if there exists $\alpha \in \mathbb{C}$ such that:

1. $\tilde{c}(x)=c(\alpha x)$

2. $\tilde{a}(x)=\alpha^{2}(a(\alpha x))$

For the sake of illustration, we provide another simple example of two diagonal metrics giving rise to isomorphic Kähler-Poisson algebras.

Example 4.14. Let $\mathcal{K}=(\mathcal{A}(x, y), g,\{x, y\})$ and $\tilde{\mathcal{K}}=(\mathcal{A}(x, y), \tilde{g},\{x, y\})$ be KählerPoisson algebras, with

$$
g=\left(\begin{array}{cc}
a(x) & 0 \\
0 & c(x)
\end{array}\right) \quad \text { and } \quad \tilde{g}=\left(\begin{array}{cc}
\alpha^{2} a(\alpha x) & 0 \\
0 & c(\alpha x)
\end{array}\right)
$$

for $a(x), c(x), \alpha \in \mathbb{C}[x]$. We will use Proposition 4.11 to show that $\mathcal{K} \cong \tilde{\mathcal{K}}$.

1. $\tilde{a}(x)=\alpha^{2} a(\alpha x)$.

2. True, since $b=\tilde{b}=0$.

3. $\tilde{c}(x)=c(\alpha x)$.

Therefore, $\mathcal{K} \cong \tilde{\mathcal{K}}$.

For instance, with $a(x)=x, c(x)=1+x+x^{2}$ and $\alpha=-2$ one finds that

$$
g=\left(\begin{array}{cc}
x & 0 \\
0 & 1+x+x^{2}
\end{array}\right) \quad \text { and } \quad \tilde{g}=\left(\begin{array}{cc}
-8 x & 0 \\
0 & 1-2 x+4 x^{2}
\end{array}\right)
$$

gives isomorphic Kähler-Poisson algebras.

In the above results, we have investigated isomorphism classes of Kähler-Poisson algebras for rational functions in two variables. With the help of our previous results on isomorphic Kähler-Poisson algebras (Proposition 4.4 and Proposition 4.6), together with Proposition 4.9, we were able to classify Kähler-Poisson structures for certain classes of metrics. Although the general case still remains open, these results give a basic insight into the moduli space problem for Kähler-Poisson algebras. 


\section{References}

[1] J. Arnlind and G. Huisken. Pseudo-Riemannian geometry in terms of multilinear brack- ets. Lett. Math. Phys., 104(12):1507-1521, 2014.

[2] J. Arnlind, J. Hoppe, and G. Huisken. Multi-linear formulation of differential geometry and matrix regularizations. J. Differential Geom., 91(1):1-39, 2012.

[3] J. Arnlind. A. Al-Shujary. Kähler-Poisson Algebras. J. Geometry and Physics., 136:156-172, 2019.

[4] A. Al-Shujary. Kähler-Poisson Algebras. Linköping Studies in Science and Technology. No.1813, 2018.

[5] A. Al-Shujary. Morphisms, direct sums and tensor products of Kähler-Poisson Algebras. arXiv:1906.04519.

[6] A. Al-Shujary. On moduli spaces of Kähler-Poisson algebras over rational functions in two variables. arXiv:1912.06696

[7] R. Berger. Géométrie algébrique de Poisson. C. R. Acad. Sci. Paris Sér. A-B, 289(11):A583-A585, 1979.

[8] J.-L. Brylinski. A differential complex for Poisson manifolds. J. Differential Geom., 28(1):93-114, 1988.

[9] K. Christian. Quantum groups. Graduate texts in mathematics; 155: 1995.

[10] S. Helgason. Differential geometry, Lie groups, and symmetric spaces, volume 34 of Graduate Studies in Mathematics. American Mathematical Society, Providence, RI, 2001.

[11] J.-C. Herz. Pseudo-algébres de Lie. I. C. R. Acad. Sci. Paris, 236:1935-1937, 1953.

[12] J. Huebschmann. Poisson cohomology and quantization. J. Reine Angew. Math., 408:57-113, 1990.

[13] J. Huebschmann. Extensions of Lie-Rinehart algebras and the Chern-Weil construction. In Higher homotopy structures in topology and mathematical physics (Poughkeepsie, NY, 1996), volume 227 of Contemp. Math., pages 145-176. Amer. Math. Soc., Provi- dence, RI, 1999.

[14] C.G.J. Jacobi, Vorlesungen über Dynamik, gehalten an der Universität zu Königsberg im Wintersemester 1842-1843 und nach einem von C.W. Borchardt ausgearbeiteten Hefte, herausgegeben von A. Clebsch, zweite revidirte Ausgabe,1884 (Chelsea, New York, 1969).

[15] H.W.E. Jung, Über ganze birationale Transformationen der Ebene, J. Reine Angew. Math. (184), pp. 161-174. 1942. 
[16] M. Kontsevich. Deformation quantization of Poisson manifolds. Lett. Math. Phys., 66(3):157-216, 2003.

[17] J. L. Koszul. Lectures on fibre bundles and differential geometry. Tata Institute of Fun- damental Research, Bombay, 1960.

[18] E. Kähler, Uber eine bemerkenswerte Hermitesche Metrik, Abh. Math. Sem. Hamburg Univ. 9 (1933), 173-186.

[19] A. Lichnerowicz. Les variétés de Poisson et leurs algébres de Lie associées. J. Differential Geometry, 12(2):253-300, 1977.

[20] S. Lie, Theorie der Transformationsgruppen, Zweiter Abschnitt, unter Mitwirkung von Prof. Dr. Friedrich Engel (Teubner, Leipzig, 1890).

[21] J. H. McKay and S. S.-S. Wang, Stuart. An elementary proof of the automorphism theorem for the polynomial ring in two variables. Journal of Pure and Applied Algebra - J PURE APPL ALG. 52. 91-102.(1988).

[22] J. Nash. The imbedding problem for Riemannian manifolds. Ann. of Math. (2), 63:20-63, 1956.

[23] E. Nelson. Tensor Analysis. Princeton University Press, Princeton, New Jersey, 1967.

[24] R. S. Palais. The cohomology of Lie rings. In Proc. Sympos. Pure Math., Vol. III, pages 130-137. American Mathematical Society, Providence, R.I., 1961.

[25] S.-D. Poisson, Sur la variation des constantes arbitraires dans les questions de mécanique, J. Ecole Polytechnique 8 (15):266-344, 1809.

[26] G. S. Rinehart. Differential forms on general commutative algebras. Trans. Amer. Math. Soc., 108:195-222, 1963.

[27] A. Weinstein. The local structure of Poisson manifolds. J. Differential Geom., 18(3):523- 557, 1983.

[28] R.O. Wells jr., Differential analysis on complex manifolds, Springer-Verlag, New York-Heidelberg-Berlin,1980. 


\section{Papers}

The papers associated with this thesis have been removed for copyright reasons. For more details about these see:

http://urn.kb.se/resolve?urn=urn:nbn:se:liu:diva-163164 
